\title{
FINITE-DIMENSIONAL REPRESENTATIONS OF MINIMAL NILPOTENT W-ALGEBRAS AND ZIGZAG ALGEBRAS
}

\author{
ALEXEY PETUKHOV
}

\begin{abstract}
Let $\mathfrak{g}$ be a simple finite-dimensional Lie algebra over an algebraically closed field $\mathbb{F}$ of characteristic 0 . We denote by $U(\mathfrak{g})$ the universal enveloping algebra of $\mathfrak{g}$. To any nilpotent element $e \in \mathfrak{g}$ one can attach an associative (and noncommutative as a general rule) algebra $\mathrm{U}(\mathfrak{g}, e)$ which is in a proper sense a "tensor factor" of $U(\mathfrak{g})$. In this article we consider the case in which $e$ belongs to the minimal nonzero nilpotent orbit of $\mathfrak{g}$. Under these assumptions $\mathrm{U}(\mathfrak{g}, e)$ was described explicitly in terms of generators and relations. One can expect that the representation theory of $\mathrm{U}(\mathfrak{g}, e)$ would be very similar to the representation theory of $U(\mathfrak{g})$. For example one can guess that the category of finite-dimensional $\mathrm{U}(\mathfrak{g}, e)$-modules is semisimple.

The goal of this article is to show that this is the case if $\mathfrak{g}$ is not simplylaced. We also show that, if $\mathfrak{g}$ is simply-laced and is not of type $A_{n}$, then the regular block of finite-dimensional $\mathrm{U}(\mathfrak{g}, e)$-modules is equivalent to the category of finite-dimensional modules of a zigzag algebra.
\end{abstract}

\section{INTRODUCTION}

Let $\mathfrak{g}$ be a simple finite-dimensional Lie algebra over an algebraically closed field $\mathbb{F}$ of characteristic 0 . We denote by $\mathrm{U}(\mathfrak{g})$ the universal enveloping algebra of $\mathfrak{g}$. To any nilpotent element $e \in \mathfrak{g}$ one can attach an associative (and noncommutative as a general rule) algebra $\mathrm{U}(\mathfrak{g}, e)$ which is in a proper sense a "tensor factor" of $\mathrm{U}(\mathfrak{g})$; see [Los2, Theorem 1.2.1], Pet, Theorem 2.1]. The notion of W-algebras can be traced back to the work $[\mathrm{Lyn}$; see also $[\mathrm{Kos}$. The modern definition of it (which is valid for all nilpotent elements) was given by A. Premet [Pr1]. It turns out that the simple finite-dimensional modules of $\mathrm{W}$-algebras are closely related to the primitive ideals of $\mathrm{U}(\mathfrak{g}, e)$; see [Los1, Conjecture 1.2.1]. The simple finitedimensional modules of $\mathrm{W}$-algebras have attracted considerable attention in the last decade; see $\mathrm{BrG}, \mathrm{Br}, \mathrm{BK}, \mathrm{Dodd}, \mathrm{LO}, \mathrm{Los} 1, \mathrm{PT}$.

In this article we focus on the case in which $e$ belongs to the minimal nonzero nilpotent orbit of $\mathfrak{g}$. Under these assumptions $\mathrm{U}(\mathfrak{g}, e)$ was described explicitly in terms of generators and relations in $\mathrm{Pr2}$, Theorem 1.1]. Moreover, a gap between primitive ideals of $\mathrm{U}(\mathfrak{g})$ and primitive ideals of $\mathrm{U}(\mathfrak{g}, e)$ is very small; see $\mathrm{Pr} 2$, Theorem 5.3]. One can guess that the representation theory of $\mathrm{U}(\mathfrak{g}, e)$ would be

Received by the editors June 3, 2017, and, in revised form, July 3, 2018.

2010 Mathematics Subject Classification. Primary 22E47, 16D50, 16D90.

Key words and phrases. Primitive ideals, self-injective modules, W-algebras, zigzag algebras.

The main result of the research presented in Sections 7, 8 was carried out at the IITP at the expense of Russian Science Foundation (project No. 14-50-00150). Other sections of this work were carried out as a part of a project on W-algebras supported by Leverhulme Trust Grant RPG-2013-293. 
very similar to the representation theory of $U(\mathfrak{g})$. For example one can guess that the category of finite-dimensional $\mathrm{U}(\mathfrak{g}, e)$-modules is semisimple.

We will show that, under the assumption that $\mathfrak{g} \neq \mathfrak{s l}(n)$, this is true if and only if $\mathfrak{g}$ is not simply-laced; see Theorems 2.1, 2.3. Moreover, we show that, if $\mathfrak{g}$ is simple simply-laced and the Dynkin diagram $\Gamma$ of $\mathfrak{g}$ is not of type $A_{n}$, then the regular block of the category of finite-dimensional modules of $\mathrm{U}(\mathfrak{g}, e)$ is equivalent to the category of finite-dimensional representations of a zigzag algebra $A(\Gamma)$ introduced by $\mathrm{R}$. Huerfano and M. Khovanov $\mathrm{HK}$ (they also provided a description of indecomposable modules of $A(\Gamma)$ ). Explicit generators and relations for these algebras are presented in the statement of Theorem 7.9

The cases of $\Gamma$ of types $C_{n}$ and $G_{2}$ were considered in Pr2, Corollary 7.1]. Therefore the semisimplicity result is new if $\mathfrak{g}$ is of type $B_{n}$ and $F_{4}$. If $\mathfrak{g} \cong \mathfrak{s l}(2)$, then $\mathrm{U}(\mathfrak{g}, e)$ is isomorphic to an algebra of polynomials in one variable. The regular block of the category of finite-dimensional representations of this polynomial algebra is quite reasonable but it has not enough projective objects. Therefore this block is not equivalent to the category of finite-dimensional modules over a finite-dimensional algebra. One can expect a similar situation if $\mathfrak{g} \cong \mathfrak{s l}(n)(n>2)$. Nevertheless, it is plausible that the category of finite-dimensional $\mathrm{U}(\mathfrak{g}, e)$-modules in this case can be described as locally nilpotent modules over a quiver with relations; see GS, Theorem 1.1] for an example of such a category. Perhaps, this can be done through a reduction to a similar question on a proper version of a proper Hecke algebra; see [BK, Theorem A].

The paper is organized as follows. In Section 2 we state the main results, i.e., Theorems 2.1, 2.3. and introduce the notation which we need to do this. In Section 3 we recall several facts on $\mathrm{W}$-algebras. In Section 4 we introduce the standard notation related to the simple Lie algebras and recall the notion of a cell in a reflection group. In Section 5 we study primitive ideals attached to the minimal nonzero nilpotent orbit of $\mathfrak{g}$. In Section 6 we recall the notion of a projective functor and several properties of it. In Section 7 we prove Theorem 2.1. In Section 8 we prove Theorem 2.3. In the appendix we write down a numerical result on maximal subalgebras which is needed in Section 8 .

\section{Notation AND The MAIN RESUlt}

Let $\mathfrak{g}$ be a simple finite-dimensional Lie algebra over an algebraically closed field $\mathbb{F}$ of characteristic 0 , let $\Gamma$ be a Dynkin diagram of $\mathfrak{g}$, and let $e \in \mathfrak{g}$ be an element of the minimal nonzero nilpotent orbit $O \subset \mathfrak{g}$. We recall that to a pair $(\mathfrak{g}, e)$ one can attach an associative algebra $\mathrm{U}(\mathfrak{g}, e)$; see [Pr2] for details. For an $\mathbb{F}$-vector space $V$ we denote by $\operatorname{dim} V$ the dimension $V$. For an ideal $I$ of an algebra $A$ we denote by $\operatorname{Dim} I$ the Gelfand-Kirillov dimension of $A / I$. For a set $S$ we denote by $|S|$ the number of elements in $S$.

We denote by $\mathrm{Z}(\mathfrak{g})$ the center of $\mathrm{U}(\mathfrak{g})$. Algebra $\mathrm{Z}(\mathfrak{g})$ can be canonically identified with the center of $\mathrm{U}(\mathfrak{g}, e)$ (see [Pr2, Corollary 5.1] and [Pr2, footnote 2] for the general case) and thus we also use notation $\mathrm{Z}(\mathfrak{g})$ to denote the center of $\mathrm{U}(\mathfrak{g}, e)$. Let $m_{0}$ be the intersection of the augmentation ideal $(\mathfrak{g})$ of $\mathrm{U}(\mathfrak{g})$ with $\mathrm{Z}(\mathfrak{g})$. It is clear that $m_{0}$ is a maximal ideal of $\mathrm{Z}(\mathfrak{g})$. For any maximal ideal $m$ of $\mathrm{Z}(\mathfrak{g})$ we denote by

$$
\mathrm{U}(\mathfrak{g}, e)-f . d \cdot \bmod ^{m}
$$


the category of finite-dimensional $\mathrm{U}(\mathfrak{g}, e)$-modules $M$ such that

$$
\forall x \in M \exists d \in \mathbb{Z}_{>0}\left(m^{d} x=0\right) .
$$

Our main results for the simply-laced Lie algebras are as follows.

Theorem 2.1. Assume that $\mathfrak{g}$ is simply-laced and is not of type $A_{n}$. Then

a) $\mathrm{U}(\mathfrak{g}, e)-f . d . m o d^{m_{0}}$ contains exactly rank $\mathfrak{g}$ simple objects,

b) $\mathrm{U}(\mathfrak{g}, e)-f . d . \mathrm{mod}^{m_{0}}$ is equivalent to the category of representations of the zigzag algebra $A(\Gamma)$; see [HK].

The result of Theorem 2.1 can be enhanced by the following proposition. To state it we use a bit more notation; see Section 4.1. (In short, there is a surjective map from a certain vector space to the maximal ideals of $\mathrm{Z}(\mathfrak{g}), \lambda \rightarrow m_{\lambda}$. Few of these $\lambda$ 's are integral and all other $\lambda$ 's are nonintegral.)

Proposition 2.2. Assume that $\mathfrak{g}$ is simply-laced and not of type $A_{n}$. If $\lambda$ is nonintegral, then the category $\mathrm{U}(\mathfrak{g}, e)-f . d . \bmod ^{m_{\lambda}}$ contains no nonzero objects.

The main result for the nonsimply-laced Lie algebras is as follows.

Theorem 2.3. Assume that $\mathfrak{g}$ is not simply-laced and is simple. Then $\mathrm{U}(\mathfrak{g}, e)-$ f.d.mod is semisimple.

Remark 2.4. Under the assumptions of Theorem 2.1 the category $\mathrm{U}(\mathfrak{g}, e)-f . d$. mod $^{m_{0}}$ has finitely many isomorphism classes of its indecomposable objects which are parametrised by the roots of $\mathfrak{g}$; see $\mathrm{HK}$, Corollary 1]. In his talk at the conference "Representation theory and symplectic singularities" in Edinburgh T. Arakawa provided a connection between these $\mathrm{W}$-algebras and a proper class of vertex algebras. Being motivated by this connection he expressed a hope that these categories $\mathrm{U}(\mathfrak{g}, e)-f . d . m o d$ are semisimple. It turns out that this is exactly the case if $\mathfrak{g}$ is not simply-laced, and if $\mathfrak{g}$ is simply-laced the situation is almost as good as he expected.

Remark 2.5. We wish to mention that $\mathrm{U}(\mathfrak{g}, e)-f . d . \bmod$ is equivalent to a subcategory of the category of $\mathfrak{g}$-modules; see Section 7 . This allows one to apply to $\mathrm{U}(\mathfrak{g}, e)-f . d . m o d$ the technique of translation functors developed in [BJ]; see also $\mathrm{BeG}$. Under the assumption that $\mathfrak{g}$ is simply-laced this shows that any block of $\mathrm{U}(\mathfrak{g}, e)-f . d . m o d$ is either semisimple with a unique simple object or is equivalent to the category of representations of the zigzag algebra $A(\Gamma)$. Key examples of semisimple blocks are given in Proposition 7.6

Remark 2.6. The dimensions of the simple finite-dimensional $\mathrm{U}(\mathfrak{g}, e)$-modules can be computed through the Goldie ranks of primitive ideals of $U(\mathfrak{g})$; see $[\mathrm{Pr} 2$, Theorem 5.3(2)]. If $\mathfrak{g}$ is not simply-laced, then this fact leads to a very explicit answer; see [Pr2, Theorem 6.2].

To prove Theorem 2.1 we use a connection between simple $\mathrm{U}(\mathfrak{g}, e)$-modules and primitive ideals of $\mathrm{U}(\mathfrak{g})$; see [Los1, Conjecture 1.2.1]. Using this approach, one can classify all simple finite-dimensional $\mathrm{U}(\mathfrak{g}, e)$-modules; see [LO].

\section{Properties of $\mathrm{U}(\mathfrak{g}, e)$}

Let $e \in \mathfrak{g}$ be a nilpotent element. For a general definition of $\mathrm{W}$-algebra $\mathrm{U}(\mathfrak{g}, e)$ see $\operatorname{Pr} 2$. Here we explore the features of this object which we need in this work. 
3.1. Skryabin's equivalence. To an element $e \in \mathfrak{g}$ one can assign (in a noncanonical way) a Lie subalgebra

$$
\mathfrak{m}(e) \subset(\mathfrak{g} \oplus \mathbb{F}) \subset \mathrm{U}(\mathfrak{g})
$$

such that the category $\mathrm{U}(\mathfrak{g}, e)-\bmod$ of $\mathrm{U}(\mathfrak{g}, e)$-modules is equivalent to the category $(\mathfrak{g}, \mathfrak{m}(e))-$ l.n.mod of $\mathfrak{g}$-modules with a locally nilpotent action of $\mathfrak{m}(e)$ (Skryabin's equivalence; see $[\mathrm{Pr} 2$ or Los1] for details). We use a particular choice of $\mathfrak{m}(e)$ defined by $[\mathrm{Pr} 2$, Subsection 2.1]. For a $\mathrm{U}(\mathfrak{g}, e)$-module $M$ we denote by $\operatorname{Skr}(M)$ the corresponding $(\mathfrak{g}, \mathfrak{m}(e))$-module. This immediately defines a map

$$
\mathcal{P}: M \rightarrow \operatorname{Ann}_{\mathrm{U}(\mathfrak{g})} \operatorname{Skr}(M)
$$

from the set of simple finite-dimensional $\mathrm{U}(\mathfrak{g}, e)$-modules to the set of primitive ideals of $\mathrm{U}(\mathfrak{g})$. The following proposition describes the image of $\mathcal{P}$ under the assumption that $O$ is the minimal nilpotent orbit of $\mathfrak{g}$ (for the general case see [LO]).

Proposition 3.1. Assume that $O$ is the minimal nonzero nilpotent orbit of $\mathfrak{g}$. Let $m$ be a maximal ideal of $\mathrm{Z}(\mathfrak{g})$. Then $\mathcal{P}$ defines a bijection between the isomorphism classes of finite-dimensional simple $\mathrm{U}(\mathfrak{g}, e)$-modules which are annihilated by $m$, and the primitive ideals $I$ of $\mathrm{U}(\mathfrak{g})$ with $I \supset m$ and $\operatorname{Var}(I)=\bar{O}$ where $\operatorname{Var}(I)$ is the associated variety of I defined in [Pr2, Subsection 3.2]; see also Subsection 4.2 ,

Proof. The proposition is equivalent to $\mathrm{Pr2}$, Theorem 5.3(5)].

3.2. Generators and relations for $\mathrm{U}(\mathfrak{g}, e)$. Denote by $\mathfrak{g}_{e}$ the centralizer of $e$ in $\mathfrak{g}$. One can consider $\mathrm{U}(\mathfrak{g}, e)$ as a deformation of the universal enveloping algebra $\mathrm{U}\left(\mathfrak{g}_{e}\right)$. Using this approach one can provide $\mathrm{U}(\mathfrak{g}, e)$ with a PBW-basis and evaluate the defining set of relations; see Pr2, Subsection 1.1]. These generators and relations are known explicitly under the assumption that $e$ belongs to the minimal nilpotent orbit $O$ as we explain next (see also [Pr2, Theorem 1.1]).

From now on $e \in O \subset \mathfrak{g}$. There are elements $h, f \in \mathfrak{g}$ such that $\{e, h, f\}$ is a standard $\mathfrak{s l}(2)$-triple. The semisimple element $h$ acts semisimply on $\mathfrak{g}$ with integral eigenvalues. We denote by $\mathfrak{g}_{e}(i)$ the respective eigenspaces. The associative algebra $\mathrm{U}(\mathfrak{g}, e)$ is generated by copies $\widetilde{\mathfrak{g}_{e}(0)}, \widetilde{\mathfrak{g}_{e}(1)}$ of $\mathfrak{g}_{e}(0), \mathfrak{g}_{e}(1)$, and central element $C$ modulo the following relations:

1) $\forall x, y \in \widetilde{\mathfrak{g}_{e}(0)}\left([x, y]=x y-y x \in \widetilde{\left.\mathfrak{g}_{e}(0)\right)}\right.$; the respective Lie bracket on $\widetilde{\mathfrak{g}_{e}(0)}$ coincides with one induced from $\mathfrak{g}_{e}(0)$,

2) $\forall x \in \widetilde{\mathfrak{g}_{e}(0)} \forall y \in \widetilde{\mathfrak{g}_{e}(1)}\left([x, y]=x y-y x \in \widetilde{\mathfrak{g}_{e}(1)}\right)$, i.e., $\widetilde{\mathfrak{g}_{e}(1)}$ is a $\widetilde{\mathfrak{g}_{e}(0)}$-module; $\widetilde{\mathfrak{g}_{e}(1)}$ is isomorphic to $\mathfrak{g}_{e}(1)$ as a $\mathfrak{g}_{e}(0)$-module,

$3)$ a formula for $[x, y]\left(x, y \in \widetilde{\mathfrak{g}_{e}(1)}\right)$; see [Pr2, Theorem 1.1].

Further we will identify $\widetilde{\mathfrak{g}_{e}(0)}$ with $\mathfrak{g}_{e}(0)$ and $\widetilde{\mathfrak{g}_{e}(1)}$ with $\mathfrak{g}_{e}(1)$.

Using these formulas one can easily check that, if $\mathfrak{g}$ is not of type $\mathrm{A}$, then $\mathrm{U}(\mathfrak{g}, e)$ has a unique one-dimensional module which is isomorphic to

$$
\mathrm{U}(\mathfrak{g}, e) / \mathrm{U}(\mathfrak{g}, e)\left(\mathfrak{g}_{e}(0) \oplus \mathfrak{g}_{e}(1)\right) ;
$$

see [Pr2, Corollary 4.1]. The following proposition is crucial for the present work.

Proposition 3.2. Assume that $\mathfrak{g}$ is not of type $A$ and that $M$ is the one-dimensional $\mathrm{U}(\mathfrak{g}, e)$-module defined above. Then $\operatorname{Ext}_{\mathrm{U}(\mathfrak{g}, e)}^{1}(M, M)=0$. 
Proof. It is enough to show that $M$ has no nontrivial self-extensions. Indeed, let

$$
0 \rightarrow M \rightarrow \tilde{M} \rightarrow M \rightarrow 0
$$

be a self-extension of $M$. Then

$$
\left(\operatorname{Ann}_{\mathrm{U}(\mathfrak{g}, e)} M\right)^{2} \subset \operatorname{Ann}_{\mathrm{U}(\mathfrak{g}, e)} \tilde{M} \subset \operatorname{Ann}_{\mathrm{U}(\mathfrak{g}, e)} M .
$$

We claim that $\operatorname{Ann}_{\mathrm{U}(\mathfrak{g})} \tilde{M}=\operatorname{Ann}_{\mathrm{U}(\mathfrak{g}, e)} M$. To show this we prove that $\operatorname{Ann}_{\mathrm{U}(\mathfrak{g}, e)} M$ $=\left(\operatorname{Ann}_{\mathrm{U}(\mathfrak{g}, e)} M\right)^{2}$.

It is easy to see that $\left[\mathfrak{g}_{e}(0), \mathfrak{g}_{e}(0)\right]=\mathfrak{g}_{e}(0)$ and $\left[\mathfrak{g}_{e}(0), \mathfrak{g}_{e}(1)\right]=\mathfrak{g}_{e}(1)$; see $[$ Pr2, Corollary 4.1]. This implies that $\operatorname{Ann}_{\mathrm{U}(\mathfrak{g}, e)} M$ is generated by $\mathfrak{g}_{e}(0)$ as a two-sided ideal. Using once more that $\left[\mathfrak{g}_{e}(0), \mathfrak{g}_{e}(0)\right]=\mathfrak{g}_{e}(0)$ we see that $\operatorname{Ann}_{\mathrm{U}(\mathfrak{g}, e)} M=$ $\left(\operatorname{Ann}_{\mathrm{U}(\mathfrak{g}, e)} M\right)^{2}$.

The claim implies that $\operatorname{Ann}_{\mathrm{U}(\mathfrak{g}, e)} \tilde{M}$ is a $\left(\mathrm{U}(\mathfrak{g}, e) / \mathrm{Ann}_{\mathrm{U}(\mathfrak{g}, e)} M\right)$-module. The fact that $M$ is one-dimensional implies that $\mathrm{U}(\mathfrak{g}, e) / \operatorname{Ann}_{\mathrm{U}(\mathfrak{g}, e)} M \cong \mathbb{F}$. Therefore $\tilde{M} \cong M \oplus M$. Thus $\operatorname{Ext}_{\mathrm{U}(\mathfrak{g}, e)}^{1}(M, M)=0$.

\section{ON THE Classification OF PRIMITIVE IDEALS OF $\mathrm{U}(\mathfrak{g})$}

We need a quite detailed description of the set of primitive ideals of $U(\mathfrak{g})$ together with the respective notation.

4.1. Notation. We assume that $\mathfrak{g}$ is a simple Lie algebra. Denote by $\mathfrak{b} \subset \mathfrak{g}$ a Borel subalgebra of $\mathfrak{g}$ and by $\mathfrak{h} \subset \mathfrak{b}$ a Cartan subalgebra of $\mathfrak{b}$. We have

$$
\mathfrak{g}=\bigoplus_{\alpha \in \mathfrak{h}^{*}} \mathfrak{g}_{\alpha}
$$

where $\mathfrak{g}_{0}=\mathfrak{h}$, $\operatorname{dim} \mathfrak{g}_{\alpha} \leq 1$ if $\alpha \neq 0$, and if $\mathfrak{g}_{\alpha} \neq 0$, then $\mathfrak{g}_{\alpha}$ is a simple onedimensional $\mathfrak{h}$-module with a character $\alpha$. We put

$$
\Delta:=\left\{0 \neq \alpha \in \mathfrak{h}^{*} \mid \mathfrak{g}_{\alpha} \neq 0\right\}, \quad \Delta^{+}:=\left\{0 \neq \alpha \in \mathfrak{h}^{*} \mid \mathfrak{g}_{\alpha} \subset \mathfrak{b}\right\}, \quad \rho:=\frac{1}{2} \sum_{\alpha \in \Delta^{+}} \alpha .
$$

We denote

- by $(\cdot, \cdot)$ the Cartan-Kiling form of $\mathfrak{g}$,

- by $\Pi$ the simple roots of $\Delta^{+}$, and by

$$
\{\omega(\alpha)\}_{\alpha \in \Pi} \subset \mathfrak{h}^{*}
$$

the corresponding fundamental weights,

- by $\Lambda$ the lattice generated by $\{\omega(\alpha)\}_{\alpha \in \Pi \text {, }}$

- by $\Lambda^{+}$the semigroup with 0 generated by $\{\omega(\alpha)\}_{\alpha \in \Pi \text {, }}$,

- by $W$ the subgroup generated by reflections with respect to the elements of $\Delta$.

Note that $(\cdot, \cdot)$ canonically identifies $\mathfrak{g}$ and $\mathfrak{g}^{*}$ and is nondegenerate after the restriction to $\mathfrak{h}$. Hence it also identifies $\mathfrak{h}$ and $\mathfrak{h}^{*}$.

Fix $\lambda \in \Lambda$. Put

$$
\Delta_{\lambda}:=\left\{\alpha \in \Delta \mid \frac{2(\lambda+\rho, \alpha)}{(\alpha, \alpha)}=0\right\} .
$$

Definition 4.1. We say that $\lambda$ is singular if $\Delta_{\lambda} \neq \emptyset$, and we say that $\lambda$ is regular otherwise. 
Definition 4.2. We say that $\lambda \in \Lambda$ is dominant if $\lambda \in \Lambda^{+}$. We say that $\lambda$ is $\rho$-dominant if $\lambda+\rho$ is dominant.

Definition 4.3. We say that two roots $\alpha, \beta \in \Pi$ are adjacent if $\alpha \neq \beta$ and $(\alpha, \beta) \neq 0$.

To any $\lambda \in \mathfrak{h}^{*}$ we assign a one-dimensional $\mathfrak{h}$-module $\mathbb{F}_{\lambda}$ which we also consider as a $\mathfrak{b}$-module $(\mathfrak{h} \cong \mathfrak{b} / \mathfrak{n}$ where $\mathfrak{n}$ is the nilpotent radical of $\mathfrak{b})$. Put

$$
M(\lambda):=\mathrm{U}(\mathfrak{g}) \otimes_{\mathrm{U}(\mathfrak{b})} \mathbb{F}_{\lambda}, \quad m_{\lambda}:=\mathrm{Z}(\mathfrak{g}) \cap \operatorname{Ann}_{\mathrm{U}(\mathfrak{g})} M(\lambda) .
$$

The ideal $m_{\lambda}$ is maximal in $\mathrm{Z}(\mathfrak{g})$ for all $\lambda$ and thus we have a map from $\mathfrak{h}^{*}$ to the set of maximal ideals of $\mathrm{Z}(\mathfrak{g})$. Moreover,

$$
m_{\lambda}=m_{\mu} \Leftrightarrow \exists w \in W(w(\lambda+\rho)=\mu+\rho) .
$$

For any $\alpha \in \Delta$ we denote by $s_{\alpha}$ the corresponding reflection.

We set $L(\lambda)$ to be the unique simple quotient of $M(\lambda)$ and $I(\lambda):=\operatorname{Ann}_{\mathrm{U}(\mathfrak{g})} L(\lambda)$. According to Duflo's theorem, for any primitive ideal $I$ of $\mathrm{U}(\mathfrak{g})$ there exists $\lambda \in \mathfrak{h}^{*}$ such that $I=I(\lambda)$. Put

$$
\tau_{L}(w):=\left\{\alpha \in \Pi \mid w^{-1} \alpha \in \Delta^{+}\right\}, \quad \tau_{R}(w):=\left\{\alpha \in \Pi \mid w \alpha \in \Delta^{+}\right\} .
$$

The following lemma provides a very useful invariant of primitive ideals.

Lemma 4.4. Fix $w_{1}, w_{2} \in W$. If $I\left(w_{1} \rho-\rho\right)=I\left(w_{2} \rho-\rho\right)$, then $\tau_{R}\left(w_{1}\right)=\tau_{R}\left(w_{2}\right)$. Thus we can define

$$
\tau(I):=\tau_{R}\left(w_{1}\right)=\tau_{R}\left(w_{2}\right) .
$$

Proof. See [BJ, Subsection 2.14]; see also [Vog, Theorem 2.4] and the text above it.

4.2. Associated varieties of ideals. The universal enveloping algebra $U(\mathfrak{g})$ of $\mathfrak{g}$ has natural degree filtration $\left\{U_{i}\right\}_{i \geq 0}$ where $U_{-1}=\{0\}$. The associated graded algebra

$$
\operatorname{gr} \mathrm{U}(\mathfrak{g}):=\bigoplus_{i \geq 0}\left(U_{i} / U_{i-1}\right)
$$

is canonically isomorphic to the symmetric algebra $S^{\cdot}(\mathfrak{g})$ of $\mathfrak{g}$. For a two-sided ideal $I$ we put

$$
\operatorname{gr} I:=\bigoplus_{i \geq 0}\left(I \cap U_{i} / I \cap U_{i-1}\right) \subset \operatorname{gr} \mathrm{U}(\mathfrak{g}) \cong \mathrm{S}^{\cdot}(\mathfrak{g}) .
$$

We put $\operatorname{Var}(I):=\left\{x \in \mathfrak{g}^{*} \mid \forall f \in \operatorname{gr} I(f(x)=0)\right\}$. It is known that if $I$ is a primitive ideal of $\mathrm{U}(\mathfrak{g})$, then $\operatorname{Var}(I)$ is the closure $\overline{O(I)}$ of a nilpotent coadjoint orbit $O(I)$ in $\mathfrak{g}^{*}$; see [Jo1]. We put $O(\lambda):=O(I(\lambda))$. Note that $O(\lambda)$ is the minimal nilpotent orbit of $\mathfrak{g}$ only for very exceptional $\lambda$ 's; see Section 5 .

4.3. One-sided and two-sided cells. Next, we need some combinatorial data attached to the reflection group $W$. It has some definition through the KazhdanLusztig polynomials (see, for example, LO, Section 6]) and it has a much more explicit description for all classical Lie algebras; see [BV]. But we believe that the approach which we use here also makes sense; cf. with $[\mathrm{BB}$. We introduce two relations on $W$ :

1) $w_{1} \sim_{L} w_{2} \Leftrightarrow I\left(w_{1} \rho-\rho\right)=I\left(w_{2} \rho-\rho\right)\left(w_{1}, w_{2} \in W\right)$,

2) $w_{1} \sim_{R} w_{2} \Leftrightarrow I\left(w_{1}^{-1} \rho-\rho\right)=I\left(w_{2}^{-1} \rho-\rho\right)\left(w_{1}, w_{2} \in W\right)$. 
Clearly, $\sim_{L}$ and $\sim_{R}$ are equivalence relations on $W$, and we denote by $\sim$ the smallest equivalence relation on $W$ which includes both $\sim_{L}, \sim_{R}$. We denote by

- $\operatorname{LCell}(w, \Pi)$ the equivalence class of $\sim_{L}$ which contains $w$,

- $\operatorname{RCell}(w, \Pi)$ the equivalence class of $\sim_{R}$ which contains $w$,

- $\operatorname{TCell}(w, \Pi)$ the equivalence class of $\sim$ which contains $w$.

It is clear that the set of left cells can be naturally identified with the set of the primitive ideals of the form $I(w \rho-\rho)(w \in W)$. The following proposition gives a straightforward connection between the associated varieties of ideals and the two-sided cells.

Proposition 4.5 ([LO, Subsection 6.2]). The two-sided ideals $I\left(w_{1} \rho-\rho\right)$ and $I\left(w_{2} \rho-\rho\right)$ have the same associated variety if and only if $w_{1} \sim w_{2}$.

Proposition 4.5 defines a map from the set of two-sided cells of $W$ to the set of nilpotent orbits. An orbit which belongs to the image of this map is called special. The minimal nilpotent orbit $O$ is special if and only if $\mathfrak{g}$ is simply-laced; see [CM].

\section{Primitive ideals $I$ for which $\operatorname{Var}(I)=\bar{O}$}

We recall that $O$ denotes the minimal nilpotent orbit of $\mathfrak{g}$. Let $\lambda \in \Lambda$ be a weight. Denote by $\operatorname{PrId}^{\lambda}(O)$ the set of primitive ideals $I$ of $\mathrm{U}(\mathfrak{g})$ such that $\operatorname{Var}(I)=\bar{O}$ and $m_{\lambda} \subset I$. Further in this section we assume that $\mathfrak{g}$ is simply-laced.

Lemma 5.1. We have $\left|\operatorname{PrId}^{0}(O)\right|=|\Pi|$.

Proof. Denote by TCell $(O)$ the two-sided cell in $W$ attached to $O$ through Proposition 4.5. The desired number of ideals equals the number of left cells in TCell $(O)$; see Subsection 4.3. According to [Jo2, Subsection 4.3], TCell $(O)$ splits into exactly $|\Pi|$ left cells.

Remark 5.2. One can deduce Lemma 5.1 from Dou].

To work out the singular integral case we need the following proposition.

Proposition 5.3 (BJ, Satz 2.14]). Fix $\lambda \in \Lambda$. Then the following sets can be identified:

1) primitive ideals $I$ of $\mathrm{U}(\mathfrak{g})$ such that $I \supset m_{\lambda}$,

2) primitive ideals $I$ of $\mathrm{U}(\mathfrak{g})$ such that $I \supset m_{0}$ and $\tau(I) \cap \Delta_{\lambda}=\emptyset$.

The associated varieties of the ideals identical under this correspondence are the same.

Using this proposition we can evaluate the desired classification of the singular cases which we need.

\section{Proposition 5.4.}

a) For $I \in \operatorname{PrId}^{0}(O)$ there exists $\alpha \in \Pi$ such that $\tau(I)=\Pi \backslash \alpha$.

b) For any $\alpha \in \Pi$ there exists a unique $I \in \operatorname{PrId}^{0}(O)$ such that $\tau(I)=\Pi \backslash \alpha$.

c) For any $\alpha \in \Pi$ there exists exactly one primitive ideal $I$ such that $I \supset$ $m_{-\omega(\alpha)}$.

Proof. Part c) is implied by parts a) and b), and Proposition 5.3. Part a) is implied by part b) and Lemma 5.1. To prove part b) we first show that for any $\alpha \in \Pi$ there exists $I \in \operatorname{PrId}^{0}(O)$ such that $\tau(I)=\Pi \backslash \alpha$. 
Denote by $\alpha_{0}$ the unique simple root with three neighbours. For any $\alpha \in \Pi$ put $\alpha_{0}, \alpha_{1}, \ldots, \alpha_{n(\alpha)}$ to be the shortest sequence of adjacent roots which connects $\alpha_{0}$ with $\alpha=\alpha_{n(\alpha)}$. Set

$$
w_{\alpha}:=s_{n(\alpha)} \ldots s_{\alpha_{1}} s_{\alpha_{0}} \in W
$$

where $s_{\alpha_{i}} \in W$ is the reflection with respect to $\alpha_{i}$. It follows from [Jo2, Subsection 4.3] that

is a left cell in $\operatorname{TCell}(O)$. Thus

$$
\left\{w_{\alpha}\right\}_{\alpha \in \Pi}
$$

$$
\left\{w_{\alpha}^{-1}\right\}_{\alpha \in \Pi}
$$

is a right cell in $\mathrm{TCell}(O)$. One can check that

$$
\tau\left(I\left(w_{\alpha}^{-1} \rho-\rho\right)\right)=\tau_{R}\left(w_{\alpha}^{-1}\right)=\tau_{L}\left(w_{\alpha}\right)=\Pi \backslash\{\alpha\} ;
$$

see also [Jo2, Subsection 4.3-4.4].

To complete the proof of part b) we mention that the ideals $I\left(w_{\alpha}^{-1} \rho-\rho\right)(\alpha \in \Pi)$ are distinct, and that according to Lemma 5.1] we have $\left|\operatorname{PrId}^{0}(O)\right|=|\Pi|$.

Proof of Theorem 2.13). It follows from a composition of Propositions 3.1, 5.4.

\section{The SEMIRING OF PROJECTIVE FUNCTORS}

Let $\lambda \in \Lambda$ be a weight and let $V$ be a finite-dimensional $\mathfrak{g}$-module. We say that a functor

$$
F: \mathrm{U}(\mathfrak{g})-\bmod ^{m_{\lambda}} \rightarrow \mathrm{U}(\mathfrak{g})-\bmod
$$

is projective if it is a direct summand of a functor

$$
\cdot \otimes V: M \rightarrow M \otimes V\left(\mathrm{U}(\mathfrak{g})-\bmod ^{m_{\lambda}} \rightarrow \mathrm{U}(\mathfrak{g})-\bmod \right)
$$

cf. with $\left[\mathrm{BeG}\right.$. It can be checked that $\cdot \otimes V$ maps $\mathrm{U}(\mathfrak{g})-\bmod ^{m_{\lambda}}$ to $\bigoplus_{\mu+\rho \in \Lambda^{+}} \mathrm{U}(\mathfrak{g})-$ $\bmod ^{m_{\mu}} \subset \mathrm{U}(\mathfrak{g})-\bmod$ and therefore one can consider projective functors as endofunctors of the category $\bigoplus_{\mu+\rho \in \Lambda^{+}} \mathrm{U}(\mathfrak{g})-\bmod ^{m_{\mu}}$.

The projective functors enjoy the following properties:

- a projective functor is exact,

- a projective functor is a direct sum of finitely many indecomposable projective functors,

- the composition of projective functors is projective,

- the direct sum of projective functors is projective;

see [BeG, Section 3]. The indecomposable projective functors can be described as follows. For any pair $\chi, \xi \in \Lambda$ one can assign an indecomposable projective functor

$$
F_{\chi, \xi}: \mathrm{U}(\mathfrak{g})-\bmod ^{m_{\chi}} \rightarrow \mathrm{U}(\mathfrak{g})-\bmod ^{m_{\xi}} .
$$

Two such functors $F_{\chi_{1}, \xi_{1}}$ and $F_{\chi_{2}, \xi_{2}}$ are isomorphic if and only if there exists $w \in W$ such that

$$
w\left(\chi_{1}+\rho\right)=\chi_{2}+\rho \text { and } w\left(\xi_{1}+\rho\right)=\xi_{2}+\rho .
$$

We will be particularly interested in the following collection of functors:

$$
\begin{gathered}
\psi_{\alpha}:=F_{0,-\omega(\alpha)}: \mathrm{U}(\mathfrak{g})-\bmod ^{m_{0}} \rightarrow \mathrm{U}(\mathfrak{g})-\bmod ^{m_{-\omega(\alpha)}}(\alpha \in \Pi), \\
\phi_{\alpha}:=F_{-\omega(\alpha), 0}: \mathrm{U}(\mathfrak{g})-\bmod ^{m_{-\omega(\alpha)}} \rightarrow \mathrm{U}(\mathfrak{g})-\bmod ^{m_{0}}(\alpha \in \Pi), \\
T_{\alpha}:=F_{0,-\alpha}=F_{0, s_{\alpha} \rho-\rho}: \mathrm{U}(\mathfrak{g})-\bmod ^{m_{0}} \rightarrow \mathrm{U}(\mathfrak{g})-\bmod ^{m_{s_{\alpha} \rho-\rho}}=\mathrm{U}(\mathfrak{g})-\bmod ^{m_{0}}(\alpha \in \Pi) .
\end{gathered}
$$

It is clear that the indecomposable projective functors form a semiring with respect to the direct sum (considered as an addition) and the composition (considered as 
a multiplication). We denote this semiring by $\mathcal{R}$. The following lemma is quite standard and is pretty straightforward.

Lemma 6.1. Let Ring $_{0}(\mathcal{R})$ be the set of pairs $\left(r_{1}, r_{2}\right) \in \mathcal{R} \times \mathcal{R}$ modulo the equivalence relation $(a, b) \sim\left(a^{\prime}, b^{\prime}\right)$ if and only if there exists $t \in \mathcal{R}$ such that $a+b^{\prime}+t=a^{\prime}+b+t$.

a) The operations $\left(r_{1}, r_{2}\right)+\left(r_{3}, r_{4}\right):=\left(r_{1}+r_{3}, r_{2}+r_{4}\right),\left(r_{1}, r_{2}\right) \cdot\left(r_{3}, r_{4}\right):=$ $\left(r_{1} r_{3}+r_{2} r_{4}, r_{1} r_{4}+r_{2} r_{3}\right)$ define a structure of a ring on $\operatorname{Ring}_{0}(\mathcal{R}) . W e$ denote this ring $\operatorname{Ring}(\mathcal{R})$. We denote by

$$
\phi_{\mathcal{R}}: \mathcal{R} \rightarrow \operatorname{Ring}(\mathcal{R})(r \rightarrow(r+r, r))
$$

the respective morphism of semirings.

b) If $R^{\prime}$ is a ring and $\phi^{\prime}: \mathcal{R} \rightarrow R^{\prime}$ is a morphism of semirings, then there exists a unique morphism of rings

$$
\psi: \operatorname{Ring}(\mathcal{R}) \rightarrow R^{\prime}
$$

such that $\phi^{\prime}:=\psi \circ \phi_{\mathcal{R}}$.

Next, we note that $\mathcal{R}$ naturally acts on the Grothendieck $K$-group of the category of $\mathfrak{g}$-modules (this is a straightforward check through the definitions of an exact functor and a $K$-group). Moreover, if $\mathcal{C}$ is a subcategory of

$$
\bigoplus_{\mu+\rho \in \Lambda^{+}} \mathrm{U}(\mathfrak{g})-\bmod ^{m_{\mu}}
$$

which is stable under $\otimes V$ for any finite-dimensional $\mathfrak{g}$-module $V$, then $\mathcal{R}$ acts on the $K$-group $K(\mathcal{C})$ of $\mathcal{C}$. The endomorphisms of $K(\mathcal{C})$ form a ring and hence we have a natural action of $\operatorname{Ring}(\mathcal{R})$ on $K(\mathcal{C})$.

6.1. Basis-dependent description of $\operatorname{Ring}(\mathcal{R})$. We recall that one can attach to a projective functor $F_{\chi, \xi}$ an endomorphism $F_{\chi, \xi}^{K}$ of a free lattice generated by $\left\{\delta_{\lambda}\right\}_{\lambda \in \Lambda}$; see [BeG, Subsection 3.4]. (This corresponds to the action of $\operatorname{Ring}(\mathcal{R})$ on the Grothendieck group $K(\mathcal{O})$ of category $\mathcal{O}$.) The assignment enjoys the following properties:

- $F_{\chi_{1}, \xi_{1}}^{K}=F_{\chi_{2}, \xi_{2}}^{K}$ implies that $F_{\chi_{1}, \xi_{1}} \cong F_{\chi_{2}, \xi_{2}}$,

- $\left(F_{\chi, \xi}\right)^{K}\left(\delta_{\lambda}\right) \neq 0$ if and only if $m_{\chi}=m_{\lambda}$,

- $F_{\chi_{1}, \xi_{1}}^{K}+F_{\chi_{2}, \xi_{2}}^{K}=\left(F_{\chi_{1}, \xi_{1}} \oplus F_{\chi_{2}, \xi_{2}}\right)^{K}$,

- $F_{\chi_{1}, \xi_{1}}^{K} F_{\chi_{2}, \xi_{2}}^{K}=\left(F_{\chi_{1}, \xi_{1}} \circ F_{\chi_{2}, \xi_{2}}\right)^{K}$,

$\left(\chi_{1}, \chi_{2}, \xi_{1}, \xi_{2}, \lambda \in \Lambda\right)$; see [BeG, Subsection 3.4]. This immediately implies that the map $(\cdot)^{K}$ defines the morphism from $\operatorname{Ring}(\mathcal{R})$ to the endomorphisms of the lattice $\bigoplus_{\lambda \in \Lambda} \mathbb{F} \delta_{\lambda}$ and that this map is injective.

Next, we mention that the lattice $\bigoplus_{\lambda \in \Lambda} \mathbb{Z} \delta_{\lambda}$ carries a $W$-action defined by the formula

$$
w \cdot \delta_{\lambda}:=\delta_{w(\lambda+\rho)-\rho}(w \in W, \lambda \in \Lambda)
$$

and $F_{\chi, \xi}^{K}$ commutes with this action of $W$. One can use this fact to provide an action of a Weyl group on the Grothendieck group of the blocks of $\mathfrak{g}$-modules; see [KZ, Theorem C.2 of Appendix].

Lemma 6.2. We have

a) $\left(\phi_{\alpha}\right)^{K}\left(\delta_{-\omega(\alpha)}\right)=\delta_{0}+\delta_{s_{\alpha} \rho-\rho}=\delta_{0}+\delta_{-\alpha}$,

b) $\left(\psi_{\alpha}\right)^{K}\left(\delta_{0}\right)=\delta_{-\omega(\alpha)}$,

c) $\left(T_{\alpha}\right)^{K}\left(\delta_{0}\right)=\delta_{0}+\delta_{-\alpha}$. 
Proof. It is easy to see that a) follows from a combination of [BeG], Subsections 1.12, 3.3, 3.4] and [Hum, Theorem 7.14(a)]; b) is implied by [BeG, 3.3, Theorem(ii)b)]; c) is a consequence of parts a) and b).

For $\lambda \in \Lambda$ denote by $I d_{\lambda}$ the operator on $\bigoplus_{\mu \in \Lambda} \delta_{\mu}$ defined by the formula

$$
I_{\lambda}\left(\delta_{\mu}\right):=\left\{\begin{array}{l}
\delta_{\mu} \text { if } \mu+\rho=w(\lambda+\rho) \text { for some } w \in W, \\
0 \text { otherwise. }
\end{array}\right.
$$

The following corollary will be very useful in the proof of Theorem 2.1.

Corollary 6.3. a) $\left(\psi_{\alpha}\right)^{K}\left(\phi_{\alpha}\right)^{K}=2 I d_{-\omega(\alpha)}$,

b) $\left(\phi_{\alpha}\right)^{K}\left(\psi_{\alpha}\right)^{K}=\left(T_{\alpha}\right)^{K}$,

c) $\left(\left(T_{\alpha}\right)^{K}\right)^{2}=2\left(T_{\alpha}\right)^{K},\left(\left(T_{\alpha}\right)^{K}-I d_{0}\right)^{2}=I d_{0}$,

d) $\left(\left(\left(T_{\alpha}\right)^{K}-I d_{0}\right)\left(\left(T_{\beta}\right)^{K}-I d_{0}\right)\right)^{3}=I d_{0}$ if $\alpha$ and $\beta$ are adjacent,

e) $\left(\left(\left(T_{\alpha}\right)^{K}-I d_{0}\right)\left(\left(T_{\beta}\right)^{K}-I d_{0}\right)\right)^{2}=I d_{0}$ if $\alpha$ and $\beta$ are not adjacent and $\alpha \neq \beta$.

\section{Proof of Theorem 2.1}

To start with we note that $\mathrm{U}(\mathfrak{g}, e)-f . d . \bmod$ is equivalent to the category $\mathcal{C}$ of $(\mathfrak{g}, \mathfrak{m}(e))$-l.n.modules of finite length and of Gelfand-Kirillov dimension $\frac{1}{2} \operatorname{dim} O$; see [Los2, Proposition 3.3.5]. We have

$$
\mathcal{C} \subset \bigoplus_{\mu+\rho \in \Lambda^{+}} \mathrm{U}(\mathfrak{g})-\bmod ^{m_{\mu}}
$$

This implies that $\operatorname{Ring}(\mathcal{R})$ acts on $K(\mathcal{C})$. For any $F \in \operatorname{Ring}(\mathcal{R})$ we denote by $(F)^{K(\mathcal{C})}$ the image of it in $\operatorname{End}(K(\mathcal{C}))$.

For any $\lambda \in \Lambda$ we put $\mathcal{C}^{\lambda}:=\mathcal{C} \cap \mathrm{U}(\mathfrak{g})-\bmod ^{m_{\lambda}}$. Functors $T_{\alpha}, \psi_{\alpha}, \phi_{\alpha}$ act on these categories as follows:

$$
\psi_{\alpha}: \mathcal{C}^{0} \rightarrow \mathcal{C}^{-\omega(\alpha)}, \quad \phi_{\alpha}: \mathcal{C}^{-\omega(\alpha)} \rightarrow \mathcal{C}^{0}, \quad T_{\alpha}: \mathcal{C}^{0} \rightarrow \mathcal{C}^{0} .
$$

We proceed with a description of $K\left(\mathcal{C}^{0}\right)$ and $K\left(\mathcal{C}^{-\omega(\alpha)}\right)$. For an object $M$ of $\mathcal{C}$ we denote by $[M]$ the image of it in $K(\mathcal{C})$. All objects of $\mathcal{C}, \mathcal{C}^{0}$ have finite length, and thus $K(\mathcal{C})$ (respectively, $K\left(\mathcal{C}^{0}\right)$ ) are generated by the images of the simple objects of $K(\mathcal{C})$ (respectively, of $K\left(\mathcal{C}^{0}\right)$ ).

Proposition 3.1 together with Proposition 5.4k) implies that $\mathcal{C}^{-\omega(\alpha)}$ has a unique simple object $M_{\alpha}^{-}$which corresponds to the unique primitive ideal $I$ of GelfandKirillov dimension $\operatorname{dim} O$ such that $I \supset m_{-\omega(\alpha)}$.

Proposition 3.1 together with Proposition 5.4 defines a bijection between simple objects of $\mathcal{C}^{0}$ and elements of $\Pi$. For any $\alpha \in \Pi$ we denote by $M_{\alpha}$ the respective simple object of $\mathcal{C}^{0}$.

We need the following lemma.

Lemma 7.1. Let $F$ be a projective functor and let $M_{1}, M_{2}$ be $\mathfrak{g}$-modules such that $\operatorname{Ann}_{\mathrm{U}(\mathfrak{g})} M_{1}=\operatorname{Ann}_{\mathrm{U}(\mathfrak{g})} M_{2}$. Then $\mathrm{Ann}_{\mathrm{U}(\mathfrak{g})} F\left(M_{1}\right)=\operatorname{Ann}_{\mathrm{U}(\mathfrak{g})} F\left(M_{2}\right)$. In particular, $F\left(M_{1}\right)=0$ if and only if $F\left(M_{2}\right)=0$.

Proof. It is implied by [Vog, Lemma 2.3].

Assembling together Lemma [7.1, [BJ], Satz 2.14], and Proposition 5.4 we prove the following lemma.

Lemma 7.2. We have $\psi_{\alpha}\left(M_{\beta}\right)=0$ if and only if $\alpha \neq \beta \in \Pi$. 
As a corollary we have that $T_{\alpha}\left(M_{\beta}\right)=0$ if and only if $\alpha \neq \beta ;\left(T_{\alpha}\right)^{K(\mathcal{C})}\left[M_{\alpha}\right]=$ $\Sigma_{\beta} c_{\alpha, \beta}\left[M_{\beta}\right]$ for some $c_{\alpha, \beta} \in \mathbb{Z}_{\geq 0}$.

Lemma 7.3. We have $c_{\alpha, \alpha}=2$ for all $\alpha \in \Pi$.

Proof. It is implied by Lemma 7.2 and formula $\left(\left(T_{\alpha}\right)^{K}\right)^{2}=2\left(T_{\alpha}\right)^{K}$.

\section{Lemma 7.4.}

a) If $\alpha, \beta \in \Pi$ are adjacent, then $c_{\alpha, \beta}=c_{\beta, \alpha}=1$.

b) If $\alpha \neq \beta \in \Pi$ are not adjacent, then $c_{\alpha, \beta}=c_{\beta, \alpha}=0$.

Proof. Part a). We have that

(1) $\left(\left(T_{\alpha}\right)^{K}-I d_{0}\right)^{K(\mathcal{C})}\left((T)_{\beta}^{K}-I d_{0}\right)^{K(\mathcal{C})}\left[M_{\alpha}\right]=-\left[M_{\alpha}\right]-c_{\alpha, \beta}\left[M_{\beta}\right]-\Sigma_{\gamma \neq \alpha, \beta} c_{\alpha, \gamma}\left[M_{\gamma}\right]$,

$$
\begin{aligned}
& \left((T)_{\alpha}^{K}-I d_{0}\right)^{K(\mathcal{C})}\left((T)_{\beta}^{K}-I d_{0}\right)^{K(\mathcal{C})}\left[M_{\beta}\right] \\
& \quad=c_{\beta, \alpha}\left[M_{\alpha}\right]+\left(c_{\beta, \alpha} c_{\alpha, \beta}-1\right)\left[M_{\beta}\right]+\Sigma_{\gamma \neq \alpha, \beta}\left(c_{\beta, \alpha} c_{\alpha, \gamma}-c_{\beta, \gamma}\right)\left[M_{\gamma}\right], \\
& \quad\left((T)_{\alpha}^{K}-I d_{0}\right)^{K(\mathcal{C})}\left((T)_{\beta}^{K}-I d_{0}\right)^{K(\mathcal{C})}\left[M_{\gamma}\right]=\left[M_{\gamma}\right](\gamma \neq \alpha, \beta) .
\end{aligned}
$$

We fix $\alpha, \beta \in \Pi$ such that $\alpha$ and $\beta$ are adjacent. Formulas (11), (2), (3) together with Corollary 6.3 ) imply that

$$
\left(\begin{array}{cc}
-1 & -c_{\alpha, \beta} \\
c_{\beta, \alpha} & c_{\alpha, \beta} c_{\beta, \alpha}-1
\end{array}\right)^{3}=\left(\begin{array}{cc}
1 & 0 \\
0 & 1
\end{array}\right) .
$$

We put

$$
X:=\left(\begin{array}{cc}
-1 & -c_{\alpha, \beta} \\
c_{\beta, \alpha} & c_{\alpha, \beta} c_{\beta, \alpha}-1
\end{array}\right) .
$$

Equation (4) implies that all eigenvalues of $X$ are roots of unity of degree 3, and therefore the trace $\operatorname{tr} X$ of $X$ equals the sum of two (not necessarily distinct) roots of unity of degree 3 . On the other hand $\operatorname{tr} X$ equals $c_{\alpha, \beta} c_{\beta, \alpha}-2$, and hence $\operatorname{tr} X$ is an integer. It can be easily checked that if such a sum is an integer, then it is equal to -1 or 2 . Thus $c_{\alpha, \beta} c_{\beta, \alpha} \in\{1,4\}$. Hence $c_{\alpha, \beta}=c_{\beta, \alpha}=1$, or $c_{\alpha, \beta}=4$ and $c_{\beta, \alpha}=1$, or $c_{\alpha, \beta}=c_{\beta, \alpha}=2$, or $c_{\alpha, \beta}=1$ and $c_{\beta, \alpha}=4$. It can be easily seen that $X^{3}=1$ if and only if $c_{\alpha, \beta}=c_{\beta, \alpha}=1$.

Part b). We fix $\alpha, \beta \in \Pi$ such that $\alpha$ and $\beta$ are adjacent. Formulas (1), (2), (3) together with Corollary $6.3 \mathrm{~d}$ ) imply that $X^{2}=\mathbf{1}$. Further we have

$$
\operatorname{det}(X)=(-1)\left(c_{\alpha, \beta} c_{\beta, \alpha}-1\right)-\left(-c_{\alpha, \beta}\right) c_{\beta, \alpha}=1 .
$$

These two facts together imply that $X=\mathbf{1}$ or $X=\mathbf{- 1}$. As a consequence we have $c_{\alpha, \beta}=c_{\beta, \alpha}=0$.

\section{Lemma 7.5.}

a) We have $\psi_{\alpha} M_{\alpha} \cong M_{\alpha}^{-}$.

b) If $\alpha, \beta \in \Pi$ are adjacent, then we have $\psi_{\beta}\left(\phi_{\alpha} M_{\alpha}^{-}\right) \cong M_{\beta}^{-}$.

Proof. Part a). Category $\mathcal{C}^{-\omega(\alpha)}$ has the unique simple object $M_{\alpha}^{-}$and thus $\left[\psi_{\alpha} M_{\alpha}\right]=c\left[M_{\alpha}^{-}\right]$for some $c \in \mathbb{Z}_{>0}$. Next,

$$
\left[T_{\alpha} M_{\alpha}\right]=\left[\phi_{\alpha} \psi_{\alpha} M_{\alpha}\right]=c\left[\phi_{\alpha} M_{\alpha}^{-}\right] .
$$

In particular, this implies that, for all $\beta \in \Pi, c_{\alpha, \beta}$ must be divisible by $c$. This together with Lemma 7.4 implies that $c \mid 1$, and therefore that $c=1$. 
Part b). It follows from part a) that $\phi_{\alpha} M_{\alpha}^{-} \cong T_{\alpha} M_{\alpha}$. According to Lemma 7.4 we have that $c_{\alpha, \beta}=1$. Therefore $\left[\psi_{\beta}\left(\phi_{\alpha} M_{\alpha}^{-}\right)\right]=\left[M_{\beta}^{-}\right]$, and we have $\psi_{\beta}\left(\phi_{\alpha} M_{\alpha}^{-}\right) \cong$ $M_{\beta}^{-}$.

Proposition 7.6. Categories $\mathcal{C}^{-\omega(\alpha)}$ are semisimple with a unique simple object.

Proof. Recall that $\alpha_{0}$ is the unique simple root with three neighbours. The statement of Proposition 7.6 for $\alpha=\alpha_{0}$ holds thanks to Proposition 3.2. Due to the fact that $\mathfrak{g}$ is simple, it is enough to show that if the statement of Proposition 7.6 holds for $\alpha \in \Pi$ and $\beta$ is adjacent to $\alpha$, then the statement of Proposition 7.6 also holds for $\beta$.

Thus we assume that $\alpha$ and $\beta$ are adjacent roots and $\mathcal{C}^{-\omega(\alpha)}$ is semisimple and has a unique up to an isomorphism simple object $M_{\alpha}^{-}$. Thanks to Proposition 5.4k) and Proposition 3.1] it is enough to show that $M_{\beta}^{-}$is projective in $\mathcal{C}^{-\omega(\beta)}$.

We have

$$
\operatorname{Hom}_{\mathrm{U}(\mathfrak{g})}\left(M_{\beta}^{-}, M\right) \cong \operatorname{Hom}_{\mathrm{U}(\mathfrak{g})}\left(\psi_{\beta} \phi_{\alpha} M_{\alpha}^{-}, M\right) \cong \operatorname{Hom}_{\mathrm{U}(\mathfrak{g})}\left(M_{\alpha}^{-}, \psi_{\alpha} \phi_{\beta} M\right)
$$

for an object $M$ of $\mathcal{C}^{-\omega(\beta)}$. Then the fact that $\psi_{\alpha}, \phi_{\beta}$ are exact immediately implies that $M_{\beta}^{-}$is projective.

Corollary 7.7. Put $P_{\alpha}:=\phi_{\alpha} M_{\alpha}^{-}$. For any object $M$ of $\mathcal{C}^{0}$ we have

$$
\operatorname{dim} \operatorname{Hom}_{\mathrm{U}(\mathfrak{g})}\left(M, P_{\alpha}\right)=\operatorname{dim} \operatorname{Hom}_{\mathrm{U}(\mathfrak{g})}\left(P_{\alpha}, M\right)
$$

and it equals the Jordan-Hölder multiplicity of $M_{\alpha}$ in $M$. In particular, $P_{\alpha}$ is both projective and injective.

Proof. Fix an object $M$ of $\mathrm{U}(\mathfrak{g})-f . d . \bmod ^{m_{0}}$. We have

$$
\begin{aligned}
& \operatorname{Hom}_{\mathrm{U}(\mathfrak{g})}\left(M_{\alpha}^{-}, \psi_{\alpha} M\right) \cong \operatorname{Hom}_{\mathrm{U}(\mathfrak{g})}\left(\phi_{\alpha} M_{\alpha}^{-}, M\right) \cong \operatorname{Hom}_{\mathrm{U}(\mathfrak{g})}\left(P_{\alpha}, M\right), \\
& \operatorname{Hom}_{\mathrm{U}(\mathfrak{g})}\left(\psi_{\alpha} M, M_{\alpha}^{-}\right) \cong \operatorname{Hom}_{\mathrm{U}(\mathfrak{g})}\left(M, \phi_{\alpha} M_{\alpha}^{-}\right) \cong \operatorname{Hom}_{\mathrm{U}(\mathfrak{g})}\left(M, P_{\alpha}\right) .
\end{aligned}
$$

The first two numbers in both rows are equal to the multiplicity of $M_{\alpha}$ in $M$ thanks to Proposition 7.6. Lemma 7.5] and Lemma 7.2

Proposition 7.8. Module $\bigoplus_{\alpha \in \Pi} P_{\alpha}$ is a faithfully projective object of $\mathcal{C}^{0}$; see Bass, Chapter II] and also [Mit, Theorm 4.1]. In particular, $\mathcal{C}^{0}$ is equivalent to the category of left finite-dimensional $\operatorname{End}_{\mathrm{U}(\mathfrak{g})}\left(\bigoplus_{\alpha \in \Pi} P_{\alpha}\right)$-modules.

Proof. All objects of $\mathcal{C}^{0}$ are of finite length and it is clear that $\operatorname{Hom}_{\mathcal{C}^{0}}\left(\bigoplus_{\alpha \in \Pi} P_{\alpha}, \cdot\right)$ is an exact functor which preserves arbitrary coproducts, i.e., direct sums in $\mathcal{C}^{0}$. This together with Bass, Chapter II, Theorem 1.3] implies that it is enough to show that $\bigoplus_{\alpha \in \Pi} P_{\alpha}$ is faithful, i.e., that for any object $M$ of $\mathcal{C}^{0}$ we have

$$
\operatorname{Hom}_{\mathrm{U}(\mathfrak{g})}\left(\bigoplus_{\alpha \in \Pi} P_{\alpha}, M\right) \neq 0 \text {. }
$$

This follows from Corollary 7.7 .

$$
\text { Put } A(\mathfrak{g}):=\operatorname{End}_{\mathrm{U}(\mathfrak{g})}\left(\bigoplus_{\alpha \in \Pi} P_{\alpha}\right) \text {. }
$$


7.1. Basis-dependent description of $A(\mathfrak{g})$. The goal of this subsection is to provide a convenient basis for the algebra $A(\mathfrak{g})$; see Theorem 7.9 , To do this we work out a complete set of linearly independent elements together with multiplication rules for the elements of this set. In short, we have $4 r-2$ basis elements where $r$ is the rank of $\mathfrak{g}$, and the product of two elements of the basis is equal to either 0 or an element of the basis. It can be easily seen from this presentation that $A(\mathfrak{g}) \cong A(\Gamma)$ where $\Gamma$ is a Dynkin diagram of $\mathfrak{g}$ and $A(\Gamma)$ is a zigzag algebra attached to $\Gamma$; see [HK. Altogether this will complete the proof of Theorem 2.1b).

Theorem 7.9. There exists a basis $\underline{\pi}_{\alpha}, \underline{\pi}_{\alpha}^{0}(\alpha \in \Pi), \underline{\varphi}_{\alpha \beta}(\alpha, \beta \in \Pi, \alpha$ and $\beta$ are adjacent) of $A(\mathfrak{g})$ such that

$$
\begin{aligned}
& \underline{\pi}_{\alpha} \underline{\pi}_{\beta}=\left\{\begin{array}{ll}
\underline{\pi}_{\alpha} & \text { if } \alpha=\beta \\
0 & \text { otherwise }
\end{array} \quad, \quad \underline{\pi}_{\alpha} \underline{\pi}_{\beta}^{0}=\underline{\pi}_{\beta}^{0} \underline{\pi}_{\alpha}=\left\{\begin{array}{ll}
\underline{\pi}_{\beta}^{0} & \text { if } \alpha=\beta \\
0 & \text { otherwise }
\end{array},\right.\right. \\
& \underline{\pi}_{\alpha} \underline{\varphi}_{\beta \gamma}=\left\{\begin{array}{l}
\underline{\varphi}_{\beta \gamma} \text { if } \alpha=\gamma \\
0 \quad \text { otherwise }
\end{array} \quad, \quad \underline{\varphi}_{\beta \gamma} \underline{\pi}_{\alpha}=\left\{\begin{array}{l}
\underline{\varphi}_{\beta \gamma} \text { if } \alpha=\beta \\
0 \quad \text { otherwise }
\end{array} \quad(\beta \text { and } \gamma \text { are adjacent }),\right.\right. \\
& \underline{\varphi}_{\gamma \tau} \underline{\varphi}_{\alpha \beta}=\left\{\begin{array}{ll}
\underline{\pi}_{\alpha}^{0} \quad \text { if } \alpha=\tau \text { and } \beta=\gamma \\
0 \quad \text { otherwise }
\end{array} \quad\left(\begin{array}{l}
\alpha \text { and } \beta \text { are adjacent } \\
\gamma \text { and } \tau \text { are adjacent }
\end{array}\right),\right. \\
& \underline{\pi}_{\alpha}^{0} \underline{\pi}_{\beta}^{0}=\underline{\pi}_{\alpha}^{0} \underline{\varphi}_{\beta \gamma}=\underline{\varphi}_{\beta \gamma} \underline{\pi}_{\alpha}^{0}=0 \quad(\beta \text { and } \gamma \text { are adjacent })
\end{aligned}
$$

for all $\alpha, \beta, \gamma, \tau \in \Pi$.

To start with we compute $\operatorname{dim} \operatorname{Hom}_{\mathrm{U}(\mathfrak{g})}\left(P_{\alpha}, P_{\beta}\right)$.

Lemma 7.10. For $\alpha, \beta \in \Pi$ we have

a) $\operatorname{dim} \operatorname{Hom}_{\mathrm{U}(\mathfrak{g})}\left(P_{\alpha}, P_{\alpha}\right)=2$,

b) $\operatorname{dim}_{\operatorname{Hom}}^{\mathrm{U}(\mathfrak{g})}\left(P_{\alpha}, P_{\beta}\right)=1$ if $\alpha$ and $\beta$ are adjacent,

c) $\operatorname{dim} \operatorname{Hom}_{\mathrm{U}(\mathfrak{g})}\left(P_{\alpha}, P_{\beta}\right)=0$ if $\alpha \neq \beta$ are not adjacent.

Proof. Is implied by Corollary 7.7, Lemma 7.3, Lemma 7.4,

Next, we fix an element $\varphi_{\alpha \beta} \in \operatorname{Hom}_{\mathrm{U}(\mathfrak{g})}\left(P_{\alpha}, P_{\beta}\right)$ for the pair of adjacent roots $\alpha, \beta$ (this element is unique up to scaling). We have that $\operatorname{dim} \operatorname{Hom}_{\mathrm{U}(\mathfrak{g})}\left(M_{\alpha}, P_{\alpha}\right)=$ $\operatorname{dim} \operatorname{Hom}_{\mathrm{U}(\mathfrak{g})}\left(P_{\alpha}, M_{\alpha}\right)=1$. Thus the composition of nonzero morphisms

$$
P_{\alpha} \rightarrow M_{\alpha} \rightarrow P_{\alpha}
$$

is unique up to scaling and we denote by $\pi_{\alpha}^{0}$ one such composition. We denote by $\pi_{\alpha}$ the identity morphism on $P_{\alpha}$.

The following proposition is a first approximation to Theorem 7.9 .

Proposition 7.11. The elements $\pi_{\alpha}, \pi_{\alpha}^{0}(\alpha \in \Pi)$ and $\varphi_{\alpha \beta}(\alpha, \beta \in \Pi, \alpha$ and $\beta$ are adjacent) are a basis of $A(\mathfrak{g})$.

Proof. It is clear that $\pi_{\alpha}, \pi_{\alpha}^{0}$ are not proportional. This together with Lemma $\left.7.10 \mathrm{a}\right)$ implies that they form a basis of $\operatorname{Hom}_{\mathrm{U}(\mathfrak{g})}\left(P_{\alpha}, P_{\alpha}\right)$. Next, Lemma 7.10 $\left.\mathrm{b}\right)$ implies that $\varphi_{\alpha \beta}$ is a basis of $\operatorname{Hom}_{\mathrm{U}(\mathfrak{g})}\left(P_{\alpha}, P_{\beta}\right)$ for a pair of adjacent roots $\alpha, \beta \in \Pi$. We are left to mention that $\operatorname{dim} \operatorname{Hom}_{\mathrm{U}(\mathfrak{g})}\left(P_{\alpha}, P_{\beta}\right)=0$ if $\alpha$ and $\beta$ are not adjacent according to Lemma $7.10 \mathrm{c}$ ).

To complete the proof of Theorem 7.9 we need to evaluate the products of the elements of the basis. 
Lemma 7.12. We have $\left(\pi_{\alpha}^{0}\right)^{2}=0$.

Proof. Corollary 7.7 implies that $P_{\alpha}$ has the unique proper maximal submodule which is precisely the kernel of $\pi_{\alpha}^{0}$. This implies that $P_{\alpha}$ is indecomposable and hence all endomorphisms of $P_{\alpha}$ are either scalar or nilpotent. The image of $\pi_{\alpha}^{0}$ is a simple submodule of $P_{\alpha}$ and therefore $\pi_{\alpha}^{0}$ is not a scalar operator on $P_{\alpha}$. Therefore $\pi_{\alpha}^{0}$ is nilpotent, and the fact that the image of $\pi_{\alpha}^{0}$ is simple implies that $\left(\pi_{\alpha}^{0}\right)^{2}=0$.

Lemma 7.13. Let $\alpha, \beta \in \Pi$ be adjacent one to each other. Then
a) $\pi_{\beta}^{0} \varphi_{\alpha \beta}=0$,
b) $\varphi_{\alpha \beta} \pi_{\alpha}^{0}=0$,
c) $\varphi_{\alpha \beta} \varphi_{\beta \alpha}$ is proportional to $\pi_{\beta}^{0}$.

Proof.

Part a). Corollary 7.7 implies that $P_{\beta}$ has the unique proper maximal submodule $\left(P_{\beta}\right)_{\text {sub }}$ which is precisely the kernel of $\pi_{\beta}^{0}$. Let $P_{\alpha \beta}$ be the image of $\varphi_{\alpha \beta}$. Either $P_{\alpha \beta} \subset\left(P_{\beta}\right)_{s u b}$ or $P_{\alpha \beta}=P_{\beta}$. In the first case we have $\pi_{\beta}^{0} \varphi_{\alpha \beta}=0$. Thus we proceed to the second case.

Assume that $P_{\alpha \beta}=P_{\beta}$. Then the fact that $P_{\beta}$ is projective implies that

$$
1=\operatorname{dim} \operatorname{Hom}_{\mathrm{U}(\mathfrak{g})}\left(P_{\beta}, P_{\alpha}\right) \geq \operatorname{dim} \operatorname{Hom}_{\mathrm{U}(\mathfrak{g})}\left(P_{\beta}, P_{\beta}\right)=2 .
$$

This is a contradiction.

Part b). Corollary 7.7 implies that $P_{\alpha}$ has the unique simple submodule which is precisely the image of $\pi_{\alpha}^{0}$ and which is isomorphic to $M_{\alpha}$. We have

$$
\operatorname{dim} \operatorname{Hom}_{\mathrm{U}(\mathfrak{g})}\left(M_{\alpha}, P_{\beta}\right)=\operatorname{dim} \operatorname{Hom}_{\mathrm{U}(\mathfrak{g})}\left(P_{\beta}, M_{\alpha}\right)=0 .
$$

Part c). First, we show that $\varphi_{\alpha \beta} \varphi_{\beta \alpha} \neq 0$. Assume to the contrary that $\varphi_{\alpha \beta} \varphi_{\beta \alpha}=$ 0 . This means that the image $P_{\beta \alpha}$ of $\varphi_{\beta \alpha}$ belongs to the kernel $\operatorname{Ker} \varphi_{\alpha \beta}$ of $\varphi_{\alpha \beta}$. Then we have a nonzero morphism

$$
P_{\alpha} / P_{\beta \alpha} \rightarrow P_{\beta}
$$

This immediately implies that the Jordan-Hölder multiplicities of $M_{\beta}$ in $P_{\beta \alpha}$ and $P_{\alpha} / P_{\beta \alpha}$ both are nonzero. Hence the Jordan-Hölder multiplicity of $M_{\beta}$ in $P_{\alpha}$ is at least 2. This is not the case.

Proposition 7.11 implies that

$$
\varphi_{\alpha \beta} \varphi_{\beta \alpha}=c_{1} \pi_{\beta}+c_{2} \pi_{\beta}^{0} .
$$

If $c_{1} \neq 0$, then the fact that $\left(\pi_{\beta}^{0}\right)^{2}=0$ implies that $\varphi_{\beta \alpha} \varphi_{\alpha \beta}$ is surjective and therefore that $\varphi_{\beta \alpha}$ is surjective. This is wrong; see case a). Therefore $\varphi_{\alpha \beta} \varphi_{\beta \alpha}=$ $c_{2} \pi_{\beta}^{0}$.

Lemma 7.14. Let $\alpha, \beta, \gamma \in \Pi$ be such that $\alpha$ and $\beta$ are adjacent, $\beta$ and $\gamma$ are adjacent, $\alpha \neq \gamma$. Then $\varphi_{\beta \gamma} \varphi_{\alpha \beta}=0$.

Proof. It is clear that $\alpha$ is not adjacent to $\gamma$. Thus $\operatorname{dim}_{\operatorname{Hom}(\mathfrak{g})}\left(P_{\alpha}, P_{\gamma}\right)=0$. Hence $\varphi_{\beta \gamma} \varphi_{\alpha \beta}=0$.

Proof of Theorem 7.9. Lemma 7.13 implies that, for a pair of adjacent roots $\alpha, \beta \in$ $\Pi$, there exists a nonzero constant $c_{\alpha, \beta}$ such that $\varphi_{\beta \alpha} \varphi_{\alpha \beta}=c_{\alpha, \beta} \pi_{\alpha}^{0}$. Denote by $\alpha_{0}$ the unique simple root with three neighbours. For any $\alpha \in \Pi$ put $\alpha_{0}, \alpha_{1}, \ldots, \alpha_{n(\alpha)}$ 
to be the shortest sequence of adjacent roots which connects $\alpha_{0}$ with $\alpha=\alpha_{n(\alpha)}$. Set

$$
\begin{aligned}
& \underline{\pi}_{\alpha}:=\pi_{\alpha}(\forall \alpha), \quad \underline{\varphi}_{\alpha_{n(\alpha)-1} \alpha}:=\varphi_{\alpha_{n(\alpha)-1} \alpha}\left(\alpha \neq \alpha_{0}\right) \\
& \underline{\pi}_{\alpha}^{0}:=\left\{\begin{array}{l}
\left(\prod_{0 \leq i<n(\alpha)} \frac{c_{\alpha_{i+1} \alpha_{i}}}{c_{\alpha_{i} \alpha_{i+1}}}\right) \pi_{\alpha}^{0} \quad \text { if } \alpha \neq \alpha_{0} \\
\pi_{\alpha}^{0} \quad \text { otherwise }
\end{array},\right. \\
& \underline{\varphi}_{\alpha_{n(\alpha)} \alpha_{n(\alpha)-1}}:=\left\{\begin{array}{ll}
\left(\prod_{0 \leq i<n(\alpha)} \frac{c_{\alpha_{i+1} \alpha_{i}}}{c_{\alpha_{i} \alpha_{i+1}}}\right) \frac{\varphi_{\left.\alpha_{n(\alpha)}\right)_{n(\alpha)-1}}}{c_{\alpha_{n(\alpha)}{ }^{\alpha} n(\alpha)-1}} & \text { if } \alpha \neq \alpha_{0} \\
\text { undefined otherwise }
\end{array} .\right.
\end{aligned}
$$

It can be easily checked through Lemmas $7.12,7.13,7.14$ that $\underline{\pi}_{\alpha}, \underline{\pi}_{\alpha}^{0}, \underline{\varphi}_{\alpha \beta}$ satisfy all conditions of Theorem 7.9 ,

\section{Proof of Theorem 2.3}

We need to expand our notation on Weyl groups, root systems, etc., to the nonintegral case. We use the notation of Section 4 . Fix $\lambda \in \mathfrak{h}^{*}$. Put

$$
\Delta^{\mathbb{Z}}:=\left\{\alpha \in \Delta \mid \frac{2(\lambda+\rho, \alpha)}{(\alpha, \alpha)} \in \mathbb{Z}\right\} .
$$

It is clear that $\Delta_{\lambda} \subset \Delta^{\mathbb{Z}}$. We denote by $W^{\mathbb{Z}}$ the subgroup of $W$ generated by the reflections with respect to elements of $\Delta^{\mathbb{Z}}$. To the root system $\Delta^{\mathbb{Z}}$ we attach a Lie algebra $\mathfrak{g}^{\mathbb{Z}}$ in such a way that $\mathfrak{h}$ is a Cartan subalgebra of $\mathfrak{g}^{\mathbb{Z}}$. We denote by $\mathfrak{b}^{\mathbb{Z}}$ the Borel subalgebra of $\mathfrak{g}^{\mathbb{Z}}$ attached to $\Delta^{\mathbb{Z}} \cap \Delta^{+}$. This allows us to define $M^{\mathbb{Z}}(\lambda), L^{\mathbb{Z}}(\lambda), I^{\mathbb{Z}}(\lambda)$, and $O^{\mathbb{Z}}(\lambda)$.

To any root $\alpha \in \Delta$ we attach a coroot $\alpha^{\vee}:=\frac{2 \alpha}{(\alpha, \alpha)} \in \mathfrak{h}^{*}$. The set of coroots $\Delta^{\vee}$ is a root system, and the Lie algebra $\mathfrak{g}^{\vee}$ attached to $\Delta^{\vee}$ is called the Langlands dual to $\mathfrak{g}$. It can be easily seen that $\mathfrak{g}^{\mathbb{Z}}$ is not necessarily isomorphic to a subalgebra of $\mathfrak{g}$, but $\left(\mathfrak{g}^{\mathbb{Z}}\right)^{\vee}$ is canonically isomorphic to the subalgebra of $\mathfrak{g}^{\vee}$ (it is defined as the sum of $\mathfrak{h}$ with the weight spaces of weights of $\left.\left(\Delta^{\mathbb{Z}}\right)^{\vee}\right)$. It is worth mentioning that if $\mathfrak{g}$ is simple, then $\mathfrak{g} \cong \mathfrak{g}^{\vee}$ if and only if $\mathfrak{g}$ is not of type $B_{n}, C_{n}(n \geq 3)$.

Definition 8.1. We say that a subalgebra $\mathfrak{k}$ of $\mathfrak{g}$ is an $r$-subalgebra if $\mathfrak{k}$ and $\mathfrak{g}$ have the same rank. By definition, $\left(\mathfrak{g}^{\mathbb{Z}}\right)^{\vee}$ is an r-subalgebra of $(\mathfrak{g})^{\vee}$.

We denote by $\operatorname{PrId}^{\lambda}$ the set primitive ideals $I$ of $\mathrm{U}(\mathfrak{g})$ such that $I \cap \mathrm{Z}(\mathfrak{g})=m_{\lambda}$. The following theorem is a slight modification of [BV2, Theorem 2.5].

Theorem 8.2. The map

$$
W^{\mathbb{Z}} \rightarrow \operatorname{PrId}^{\lambda} \quad(w \rightarrow I(w(\lambda+\rho)-\rho))
$$

is surjective.

Put $\rho^{\mathbb{Z}}:=\frac{1}{2} \sum_{\alpha \in \Delta^{\mathbb{Z}} \cap \Delta^{+}} \alpha$. We say that $\lambda$ is dominant with respect to $\Delta^{\mathbb{Z}} \cap \Delta^{+}$ if $\left(\lambda, \alpha^{\vee}\right) \in \mathbb{Z}_{\geq 0}$ for all $\alpha \in \Delta^{\mathbb{Z}} \cap \Delta^{+}$. The following statement is a straightforward corollary of [BV2, Theorem 4.8, Proposition 2.28].

Theorem 8.3. We have

$$
\operatorname{dim} \mathfrak{g}-\operatorname{dim} O(\lambda)=\operatorname{dim} \mathfrak{g}^{\mathbb{Z}}-\operatorname{dim} O^{\mathbb{Z}}\left(\lambda+\rho-\rho^{\mathbb{Z}}\right) .
$$


This theorem allows us to expand Theorem 2.1 beyond the integral case. Recall that

$$
\operatorname{PrId}^{\lambda}(O)=\left\{I \in \operatorname{PrId}^{\lambda} \mid \operatorname{Var}(I)=\bar{O}\right\} .
$$

Recall that $\mathcal{O}$ is the minimal nilpotent orbit of $\mathfrak{g}$. Proposition 2.2 is implied by Proposition 3.1 and the following statement.

Proposition 8.4. Assume that $\mathfrak{g}$ is simply-laced and not of type $A_{n}$. If $\lambda$ is nonintegral, then $\operatorname{PrId}^{\lambda}(O)$ is empty.

Proof. Assume to the contrary that $\lambda$ is nonintegral, and there exists an ideal $I$ in $\operatorname{PrId}^{\lambda}(O)$. Without loss of generality we can assume that $I=I(\lambda)$ and $O=O(\lambda)$.

The assumptions on $\lambda$ imply that

$$
\operatorname{dim} \mathfrak{g}^{\mathbb{Z}}<\operatorname{dim} \mathfrak{g} .
$$

Theorem 8.3 implies that

$$
\operatorname{dim} O=\operatorname{dim} \mathfrak{g}-\operatorname{dim} \mathfrak{g}^{\mathbb{Z}}+\operatorname{dim} O^{\mathbb{Z}}\left(\lambda+\rho-\rho^{\mathbb{Z}}\right),
$$

and thus

$$
\operatorname{dim} O \geq \operatorname{dim} \mathfrak{g}-\operatorname{dim} \mathfrak{g}^{\mathbb{Z}} .
$$

The dimensions of $\operatorname{dim} O$ are written in Table 1 (they are evaluated through $\mathrm{CM}$, Lemma 4.3.5]).

TABLE 1

\begin{tabular}{|c|c|c|c|c|c|c|c|c|}
\hline$A_{n}(n \geq 2)$ & $B_{n}(n \geq 2)$ & $C_{n}(n \geq 2)$ & $D_{n}(n \geq 4)$ & $E_{6}$ & $E_{7}$ & $E_{8}$ & $F_{4}$ & $G_{2}$ \\
\hline $2 n$ & $4 n-4$ & $2 n$ & $4 n-6$ & 22 & 34 & 58 & 16 & 6 \\
\hline
\end{tabular}

Comparing these numbers with Proposition 9.1 of the appendix applied to $\left(\mathfrak{g}^{\mathbb{Z}}\right)^{\vee}$ $\subset(\mathfrak{g})^{\vee}$ we see that

$$
\operatorname{dim} O<\operatorname{dim}(\mathfrak{g})^{\vee}-\operatorname{dim}\left(\mathfrak{g}^{\mathbb{Z}}\right)^{\vee}
$$

due to the fact that $\mathfrak{g}$ is simply-laced and not of type $A$. This is a contradiction.

We wish to mention that cases $C_{n}, G_{2}$ were considered in [Pr2, Corollary 7.1]. Theorem 2.3 is implied by the following proposition.

Proposition 8.5. Assume that $\mathfrak{g}$ is not simply-laced and is simple. Then the following statements are equivalent:

a) $\mathrm{U}(\mathfrak{g}, e)-$ f.d.mod $m_{\lambda}$ has a nonzero object,

b) $\mathrm{U}(\mathfrak{g}, e)-f . d . m o d^{m_{\lambda}}$ is semisimple with a unique simple object.

Proof of Proposition 8.5. It is enough to show that a) implies b). From now on we assume a). We need the following lemmas.

Lemma 8.6. Assume that $O(\lambda)=O$. Then

a) $\left(\mathfrak{g}^{\mathbb{Z}}\right)^{\vee}$ is a maximal proper $r$-subalgebra of $\mathfrak{g}^{\vee}$,

b) $\lambda+\rho-\rho^{\mathbb{Z}}$ is dominant with respect to $\Delta^{\mathbb{Z}} \cap \Delta^{+}$,

c) $W_{\lambda}=\{e\}$. 
Proof. The minimal nilpotent orbit $O$ is not special under the assumption that $\mathfrak{g}$ is not simply-laced; see [CM]. Thus $\left(\mathfrak{g}^{\mathbb{Z}}\right)^{\vee}$ is a proper r-subalgebra of $\mathfrak{g}^{\vee}$. Theorem 8.3 implies that

$$
\operatorname{dim} O=\operatorname{dim} \mathfrak{g}-\operatorname{dim} \mathfrak{g}^{\mathbb{Z}}+\operatorname{dim} O^{\mathbb{Z}}\left(\lambda+\rho-\rho^{\mathbb{Z}}\right),
$$

and thus that

$$
\operatorname{dim} O \geq \operatorname{dim} \mathfrak{g}-\operatorname{dim} \mathfrak{g}^{\mathbb{Z}} .
$$

The dimensions of $\operatorname{dim} O$ are written in Table 1 Comparing these numbers with Proposition 9.1 of the appendix applied to $\left(\mathfrak{g}^{\mathbb{Z}}\right)^{\vee} \subset(\mathfrak{g})^{\vee}$ we see that

$$
\operatorname{dim} O \leq \operatorname{dim}(\mathfrak{g})^{\vee}-\operatorname{dim}\left(\mathfrak{g}^{\mathbb{Z}}\right)^{\vee}
$$

due to the fact that $\mathfrak{g}$ is not simply-laced. Therefore Proposition 9.1 and the fact that $\left(\mathfrak{g}^{\mathbb{Z}}\right)^{\vee}$ is an r-subalgebra of $\mathfrak{g}^{\vee}$, implies that $\left(\mathfrak{g}^{\mathbb{Z}}\right)^{\vee}$ is a maximal r-subalgebra of $\mathfrak{g}^{\vee}$. Also we have that

$$
\operatorname{dim} O^{\mathbb{Z}}\left(\lambda+\rho-\rho^{\mathbb{Z}}\right)=0,
$$

and thus that $\lambda+\rho-\rho^{\mathbb{Z}}$ is dominant with respect to $\Delta^{\mathbb{Z}}$. The latter condition implies that $W_{\lambda}$ is trivial. This completes the proof.

The lemma below is well known, and we provide the proof of it only for the convenience of the reader.

Lemma 8.7. Let $\Delta_{1}, \Delta_{2} \subset \Delta$ be subsets of $\Delta$ such that

$$
\mathfrak{g}_{1}:=\mathfrak{h} \bigoplus_{\alpha \in \Delta_{1}} \mathfrak{g}_{\alpha}, \quad \mathfrak{g}_{2}:=\mathfrak{h} \bigoplus_{\alpha \in \Delta_{1}} \mathfrak{g}_{\alpha},
$$

are r-subalgebras of $\mathfrak{g}$. Then the following conditions are equivalent:

a) $\mathfrak{g}_{1}$ is conjugate to $\mathfrak{g}_{2}$ by the adjoint group $\operatorname{Adj}(\mathfrak{g})$ of $\mathfrak{g}$,

b) there exists $w \in W$ such that $w\left(\Delta_{1}\right)=\Delta_{2}$.

Proof. First, we show that a) implies b). We fix $g \in \operatorname{Adj}(\mathfrak{g})$ such that $g\left(\mathfrak{g}_{1}\right)=\mathfrak{g}_{2}$. By definition, $\mathfrak{h}$ is a Cartan subalgebra of both $\mathfrak{g}_{1}$ and $\mathfrak{g}_{2}$. Thus $g(\mathfrak{h})$ is a Cartan subalgebra of $\mathfrak{g}_{2}$. Therefore there exists an element $g_{2}$ of the adjoint group $\operatorname{Adj}\left(\mathfrak{g}_{2}\right)$ of $\mathfrak{g}_{2}$ such that $g_{2}(g(\mathfrak{h}))=\mathfrak{h}$. Put $g^{\prime}:=g_{2} g$. We have

$$
g^{\prime}\left(\mathfrak{g}_{1}\right)=\mathfrak{g}_{2}, \quad g^{\prime}(\mathfrak{h})=\mathfrak{h} .
$$

The second condition implies that $g^{\prime}$ can be represented by an element $w \in W$ and the first condition implies that this $w$ is as desired in b).

Next, we show that b) implies a). The Weyl group can be identified with the quotient of the normalizer of $\mathfrak{h}$ in $\operatorname{Adj}(\mathfrak{g})$ by the centralizer of $\mathfrak{h}$ in $\operatorname{Adj}(\mathfrak{g})$. Thus there exists $g \in \operatorname{Adj}(\mathfrak{g})$ such that

$$
g(\mathfrak{h})=\mathfrak{h}, \quad g\left(\mathfrak{g}_{\alpha}\right)=\mathfrak{g}_{w(\alpha)} .
$$

It is clear that $g\left(\mathfrak{g}_{1}\right)=\mathfrak{g}_{2}$.

Lemma 8.8. Assume that $\mathrm{U}(\mathfrak{g}, e)-f . d . \bmod ^{m_{\lambda}}$ has a nonzero object. Then $\mathrm{U}(\mathfrak{g}, e)-$ f.d.mod ${ }^{m_{\lambda}}$ has a unique simple object $M_{f . d .}(\lambda)$. Moreover, there exists $w \in W^{\mathbb{Z}}$ such that $M_{f . d .}(\lambda)=\mathcal{P}^{-1}(I(w(\lambda+\rho)-\rho))$. 
Proof. Theorem 8.2 together with Proposition 3.1 implies that any simple object of $\mathrm{U}(\mathfrak{g}, e)-f . d . \bmod ^{m_{\lambda}}$ is of the form $\mathcal{P}^{-1}(I(w(\lambda+\rho)-\rho))$ for $w \in W^{\mathbb{Z}}$.

We consider $w \in W^{\mathbb{Z}}$ such that $\mathcal{P}^{-1}(I(w(\lambda+\rho)-\rho))$ belongs to $\mathrm{U}(\mathfrak{g}, e)-$ $f . d . m o d^{m_{\lambda}}$. The latter condition is equivalent to $O(w(\lambda+\rho)-\rho)=O$. This implies that $w(\lambda+\rho)-\rho^{\mathbb{Z}}$ is dominant with respect to $\Delta^{\mathbb{Z}} \cap \Delta^{+}$. This implies that $w$ is unique. Thus we have shown that $\mathrm{U}(\mathfrak{g}, e)-f . d . \bmod ^{m_{\lambda}}$ has a unique simple object. We denote this object by $M_{f . d .}(\lambda)$.

We are left to prove that $\operatorname{Ext}^{1}\left(M_{f . d .}(\lambda), M_{f . d .}(\lambda)\right)$. If $\mathfrak{g} \cong G_{2}$ or $\mathfrak{g} \cong C_{n}$, then the statement of Proposition 8.5 follows immediately from [Pr2, Corollary 7.1]. From now on we assume that $\mathfrak{g}$ is not of type $C_{n}, G_{2}$, i.e., that $\mathfrak{g}$ is of type $B_{n}, F_{4}$. We use notation for roots and weights of [Bou], and consider both cases simultaneously. The underlying calculations can be easily done through Bou .

Set $\mu_{0}$ as in [Jo2, Table 3]. Put

$$
\Delta(\mathfrak{k}):=\left\{\alpha \in \Delta \mid\left(\alpha^{\vee}, \mu_{0}\right) \in \mathbb{Z}\right\}, \quad \mathfrak{k}^{\vee}:=\mathfrak{h} \bigoplus_{\alpha \in \Delta(\mathfrak{k})}(\mathfrak{g})_{\alpha^{\vee}}^{\vee}
$$

Dimension arguments together with Proposition 9.1 imply that $\mathfrak{k}^{\vee}$ is a maximal r-subalgebra of $\mathfrak{g}^{\vee}$. We have that $\left(\mathfrak{g}^{\mathbb{Z}}\right)^{\vee}$ is also a maximal r-subalgebra of $\mathfrak{g}^{\vee}$. Lemma 8.7 implies that there exists $w^{\prime} \in W$ such that $w^{\prime}\left(\Delta^{\mathbb{Z}}\right)=\Delta(\mathfrak{k})$. Put $\lambda^{\prime}:=w^{\prime}(\lambda+\rho)-\rho$. We have that $\mathrm{U}(\mathfrak{g}, e)-f . d . \bmod ^{m_{\lambda}}=\mathrm{U}(\mathfrak{g}, e)-f . d$. mod $^{m_{\lambda^{\prime}}}$. Thus we can assume that $\lambda=\lambda^{\prime}$. This immediately implies that $\Delta^{\mathbb{Z}}=\Delta(\mathfrak{k})$.

Let $\gamma \in \Pi$ be the unique simple root such that $\left(\gamma, \mu_{0}\right) \neq 0$ (in both cases $\gamma=\alpha_{1}$ ). Then $\Pi \backslash \gamma \in \Delta^{\mathbb{Z}}$.

It is easy to check that $2 \gamma^{\vee}$ belongs to the lattice generated by $\left(\Delta^{\mathbb{Z}}\right)^{\vee}$. Thus

$$
2\left(\gamma^{\vee}, \lambda\right) \in \mathbb{Z}
$$

Therefore either $\left(\gamma^{\vee}, \lambda\right) \in \mathbb{Z}$ or $\left(\gamma^{\vee}, \lambda\right) \in \frac{1}{2}+\mathbb{Z}$. We have that $\left(\alpha^{\vee}, \lambda\right) \in \mathbb{Z}$ for all $\alpha \in(\Pi \backslash \gamma) \subset \Delta^{\mathbb{Z}}$. Thus if $\left(\gamma^{\vee}, \lambda\right) \in \mathbb{Z}$, then $\Pi \subset \Delta^{\mathbb{Z}}$, and hence $\Delta^{\mathbb{Z}}=\Delta$. This is a contradiction. that

Thus $\left(\gamma^{\vee}, \lambda\right) \in \frac{1}{2}+\mathbb{Z}$. The fact that $\left(\alpha^{\vee}, \lambda\right) \in \mathbb{Z}$ for all $\alpha \in(\Pi \backslash \gamma) \subset \Delta^{\mathbb{Z}}$ implies

$$
\left(\lambda-\mu_{0}, \alpha^{\vee}\right) \in \mathbb{Z}
$$

As a consequence $\lambda-\mu_{0} \in \Lambda, \lambda-\mu_{0}+\rho \in \Lambda$. Lemma 8.6 implies that $\lambda+\rho-\rho^{\mathbb{Z}}$ is dominant with respect to $\Delta^{\mathbb{Z}} \cap \Delta^{+}$. It is straightforward to check that $\mu_{0}-\rho^{\mathbb{Z}}$ is dominant with respect to $\Delta^{\mathbb{Z}} \cap \Delta^{+}$.

To finish the proof we need the following lemma.

Lemma 8.9. Assume that

a) both $\lambda+\rho-\rho^{\mathbb{Z}}$ and $\mu_{0}-\rho^{\mathbb{Z}}$ are dominant with respect to $\Delta^{\mathbb{Z}} \cap \Delta^{+}$,

b) $\lambda-\mu_{0}+\rho \in \Lambda$,

c) $W_{\lambda}=W_{\mu_{0}}$.

Then $\mathrm{U}(\mathfrak{g}, e)-$ f.d.mod ${ }^{m_{\lambda}}$ is equivalent to $\mathrm{U}(\mathfrak{g}, e)-$ f.d.mod ${ }^{m_{\mu_{0}-\rho}}$.

Proof. We recall that $\mathrm{U}(\mathfrak{g}, e)-f . d . \bmod ^{m_{\lambda}}$ is equivalent to a category of $\mathfrak{g}$-modules $\mathcal{C}^{\lambda}$; see Section 7. Conditions a), b), c) imply together with BeG, Theorem, Section 4.1] that $\mathcal{C}^{\lambda}$ is equivalent to $\mathcal{C}^{\mu_{0}-\rho}$. Hence $\mathrm{U}(\mathfrak{g}, e)-f . d \cdot \bmod ^{m_{\lambda}}$ is equivalent to $\mathrm{U}(\mathfrak{g}, e)-f . d . \bmod ^{m_{\mu_{0}-\rho}}$. 
Thanks to Lemmas 8.8, 8.9 we have that

$$
\operatorname{dim} \operatorname{Ext}^{1}\left(M_{f . d .}(\lambda), M_{f . d .}(\lambda)\right)=\operatorname{dim} \operatorname{Ext}^{1}\left(M_{f . d .}\left(\mu_{0}-\rho\right), M_{f . d .}\left(\mu_{0}-\rho\right)\right) .
$$

Thus we are left to show that $\operatorname{dim} \operatorname{Ext}^{1}(M, M)=0$ for one nonzero simple finitedimensional $\mathrm{U}(\mathfrak{g}, e)$-module $M$. This was done in Proposition 3.2 .

\section{Appendix: Maximal Reductive root subalgebras}

Let $\mathfrak{g}$ be a reductive Lie algebra. By definition, the rank of $\mathfrak{g}$ equals the dimension of a Cartan subalgebra of $\mathfrak{g}$. We say that a subalgebra $\mathfrak{k}$ of $\mathfrak{g}$ is an $r$-subalgebra if the rank of $\mathfrak{k}$ equals the rank of $\mathfrak{g}$. The description of maximal by inclusion $\mathrm{r}$-subalgebras of simple Lie algebras are very well known; see [BS] and also [Bou, [Dyn. We used a description of maximal by dimension r-subalgebras in Section 8 Of course, the second one is a straightforward consequence of the first one, but this consequence requires plenty of not very conceptual computations. We decided to provide these computations in this appendix.

Proposition 9.1. Let $\mathfrak{g}$ be a simple Lie algebra. Then the conjugacy classes of maximal by dimension proper $r$-subalgebras $\mathfrak{k}$ of $\mathfrak{g}$ are listed in Table 2. In particular, such a conjugacy class is unique if $\mathfrak{g} \neq D_{4}$.

\section{TABLE 2}

\begin{tabular}{|c|c|c|c|}
\hline $\mathfrak{g}$ & $\mathfrak{k}$ & $\operatorname{codim}(\mathfrak{g} / \mathfrak{k})$ & \\
\hline$A_{l}$ & $A_{l-1} \oplus \mathbb{F}$ & $2 l-2$ & $l \geq 1$ \\
$B_{l}$ & $D_{l}$ & $2 l$ & $l \geq 2$ \\
$C_{l}$ & $C_{l-1} \oplus C_{1}$ & $4 l-4$ & $l \geq 2$ \\
$D_{l}$ & $D_{l-1} \oplus \mathbb{F}$ & $4 l-4$ & $l \geq 5$ \\
$D_{4}$ & $D_{3} \oplus \mathbb{F}, A_{3} \oplus \mathbb{F}, A_{3}^{\prime} \oplus \mathbb{F}$ & 12 & \\
$E_{6}$ & $D_{5} \oplus \mathbb{F}$ & 32 & \\
$E_{7}$ & $E_{6} \oplus \mathbb{F}$ & 54 & \\
$E_{8}$ & $E_{7} \oplus A_{1}$ & 112 & \\
$F_{4}$ & $B_{4}$ & 16 & \\
$G_{2}$ & $A_{2}$ & 6 & \\
\hline
\end{tabular}

Proof. The conjugacy classes of such subalgebras can be identified with the conjugacy classes of subgroups of maximal rank of the compact group attached to the root system of $\mathfrak{g}$. The latter conjugacy classes are described in [BS, Table]. We reproduce the needed part of [BS, Table] in Table 3 .

We are left to figure out which subalgebras of this list are maximal by dimension. For the exceptional groups this is very straightforward because the list of maximal by inclusion subalgebras is finite. We proceed with the classical cases one-by-one.

Case A. We claim that $A_{l-1} \oplus \mathbb{F}$ has the maximal dimension in $A_{l}$, and thus that

$$
\operatorname{dim}\left(A_{l-1} \oplus \mathbb{F}\right)>\operatorname{dim}\left(A_{i} \oplus A_{l-i-1} \oplus \mathbb{F}\right)
$$

if $0<i<l-1$. This is equivalent to the following statements:

$$
\begin{aligned}
l^{2}> & (i+1)^{2}+(l-i)^{2}-1, \\
& 2 l i-2 i^{2}-2 i \geq 0, \quad(0<i<l-1), \\
& 2 i(l-i-1) \geq 0 .
\end{aligned}
$$

The latter statement is trivial. 
TABLE 3

\begin{tabular}{|c|c|c|}
\hline $\mathfrak{g}$ & $\mathfrak{k}$ & \\
\hline$A_{l}$ & $A_{i} \oplus A_{l-i-1} \oplus \mathbb{F}$ & $1 \leq i<l, l \geq 1$ \\
\hline$B_{l}$ & $D_{l}$ & $l \geq 2$ \\
& $B_{i} \oplus D_{l-i}$ & $1 \leq i<l, l \geq 2$ \\
& $B_{l-1} \oplus \mathbb{F}$ & $j \geq 2$ \\
\hline$C_{l}$ & $C_{i} \oplus C_{l-i}$ & $1 \leq i<l, l \geq 2$ \\
& $A_{l-1} \oplus \mathbb{F}$ & $l \geq 2$ \\
\hline$D_{l}$ & $D_{i} \oplus D_{l-i}$ & $1 \leq i \leq l, l \geq 4$ \\
& $A_{l-1} \oplus \mathbb{F}$ & $l \geq 4$ \\
& $D_{l-1} \oplus \mathbb{F}$ & $l \geq 4$ \\
\hline$E_{6}$ & $A_{1} \oplus A_{5}, A_{2} \oplus A_{2} \oplus A_{2}$ & \\
& $D_{5} \oplus \mathbb{F}$ & \\
\hline$E_{7}$ & $A_{1} \oplus D_{6}, A_{7}, A_{2} \oplus A_{5}$ & \\
& $E_{6} \oplus \mathbb{F}$ & \\
\hline$E_{8}$ & $D_{8}, A_{1} \oplus E_{7}, A_{8}$ & \\
& $A_{2} \oplus E_{6}, A_{4} \oplus A_{4}$ & \\
\hline$F_{4}$ & $A_{1} \oplus C_{3}, B_{4}, A_{2} \oplus A_{2}$ & \\
\hline$G_{2}$ & $A_{1} \oplus A_{1}, A_{2}$ & \\
\hline \multicolumn{3}{|c|}{} \\
\hline
\end{tabular}

Case B. We claim that $D_{l}$ has the maximal dimension in $B_{l}$, and thus that

$$
\begin{array}{ccc}
\text { (B1) } & \operatorname{dim} D_{l}>\operatorname{dim}\left(B_{i} \oplus D_{l-i}\right), & 0<i<l, l \geq 3, \\
\text { (B2) } & \operatorname{dim} D_{l}>\operatorname{dim}\left(B_{l-1} \oplus \mathbb{F}\right), & l \geq 3 .
\end{array}
$$

We now verify statements (B1) and (B2).

Statement (B1) is equivalent to the following inequalities

$$
\begin{array}{ll}
2 l^{2}-l>\left(2 i^{2}+i\right)+\left(2(l-i)^{2}-(l-i)\right), & \\
2 i(2 l-i)-i>2 i^{2}+i, & (0<i<l), \\
62 i(2 l-2 i-1)>0 . &
\end{array}
$$

The latter statement is trivial.

Statement (B2) is equivalent to the following inequalities:

$$
\begin{aligned}
& 2 l^{2}-l>\left(2(l-1)^{2}+(l-1)\right)+1, \quad(l \geq 2), \\
& 2(l-1)>0 .
\end{aligned}
$$

The latter statement is trivial.

Case $\mathrm{C}$. We claim that $C_{l-1} \oplus C_{1}$ has the maximal dimension in $C_{l}$, and thus that

$$
\begin{array}{ccc}
\text { (C1) } & \operatorname{dim}\left(C_{l-1} \oplus C_{1}\right)>\operatorname{dim}\left(C_{i} \oplus C_{l-i}\right), & 1<i<l-1, l \geq 2, \\
\text { (C2) } & \operatorname{dim}\left(C_{l-1} \oplus C_{1}\right)>\operatorname{dim}\left(A_{l-1} \oplus \mathbb{F}\right), & l \geq 2 .
\end{array}
$$

We now verify statements (C1) and (C2).

Statement $(\mathrm{C} 1)$ is equivalent to the following inequalities:

$$
\begin{array}{ll}
2(l-1)^{2}+(l-1)+3>\left(2 i^{2}+i\right)+\left(2(l-i)^{2}-(l-i)\right), & \\
2(i-1)(2 l-i-1)-2(i-1)(i+1)>0, & (1<i<l-1), \\
& 4(i-1)(l-i-1)>0 .
\end{array}
$$

The latter statement is trivial. 
Statement $(\mathrm{C} 2)$ is equivalent to the following inequalities:

$$
\begin{aligned}
& 2(l-1)^{2}+(l-1)+3>l^{2}, \quad(l \geq 2), \\
& (l-2)^{2}+l>0 .
\end{aligned}
$$

The latter statement is trivial.

Case D. We claim that $D_{l-1} \oplus \mathbb{F}$ has the maximal dimension in $D_{l}$, and thus that

(D1) $\operatorname{dim}\left(D_{l-1} \oplus \mathbb{F}\right)>\operatorname{dim}\left(D_{i} \oplus D_{l-i}\right), \quad 1<i<l-1, l \geq 4$,

(D2) $\quad \operatorname{dim}\left(D_{l-1} \oplus \mathbb{F}\right)>\operatorname{dim}\left(A_{l-1} \oplus \mathbb{F}\right), \quad l \geq 4$.

We now verify statements (D1) and (D2).

Statement (D1) is equivalent to the following inequalities:

$$
\begin{aligned}
& 2(l-1)^{2}-(l-1)+1>\left(2 i^{2}-i\right)+\left(2(l-i)^{2}-(l-i)\right), \\
& 2(i-1)(2 l-i-1)-(i-1)-2(i-1)(i+1)+(i-1)>0 . \quad(1<i<l-1),
\end{aligned}
$$

The latter statement is trivial.

Statement (D2) is equivalent to the following inequalities:

$$
\begin{aligned}
& 2(l-1)^{2}-(l-1)+1>l^{2}, \quad(l \geq 4) . \\
& (l-1)(l-4)>0,
\end{aligned}
$$

If $l \geq 5$ the latter statement is trivial. If $l=4$, then $D_{3}=A_{3}$ and the subalgebras having the same dimension $D_{3} \oplus \mathbb{F}, A_{3} \oplus \mathbb{F}$, and $A_{3}^{\prime} \oplus \mathbb{F}$ are not conjugate in $D_{4}$.

\section{ACKNOWLEDGMENTS}

The author would like to thank Alexander Premet for many useful comments on this article, and many stimulating questions on the previous versions of it. The author would like to thank Tobias Kildetoft for the reference [Dou, Mike Prest for the reference [Mit, Catharina Stroppel for the reference [HK], Lewis Topley for the reference [Los2], and Érnest Vinberg for the reference [BS]. The author is grateful to the referee for a very careful reading of the previous version of this article, and for providing a huge amount of very detailed (and very helpful) comments.

\section{REFERENCES}

[Bass] Hyman Bass, Algebraic K-theory, W. A. Benjamin, Inc., New York-Amsterdam, 1968. MR.0249491

[BV] Dan Barbasch and David Vogan, Primitive ideals and orbital integrals in complex classical groups, Math. Ann. 259 (1982), no. 2, 153-199, DOI 10.1007/BF01457308. MR656661

[BV2] Dan Barbasch and David Vogan, Primitive ideals and orbital integrals in complex exceptional groups, J. Algebra 80 (1983), no. 2, 350-382, DOI 10.1016/0021-8693(83)90006-6. MR691809

[BeG] J. N. Bernstein and S. I. Gel'fand, Tensor products of finite- and infinite-dimensional representations of semisimple Lie algebras, Compositio Math. 41 (1980), no. 2, 245-285. MR.581584

[BS] A. Borel and J. De Siebenthal, Les sous-groupes fermés de rang maximum des groupes de Lie clos (French), Comment. Math. Helv. 23 (1949), 200-221, DOI 10.1007/BF02565599. MR 0032659

[BB] W. Borho and J.-L. Brylinski, Differential operators on homogeneous spaces. III. Characteristic varieties of Harish-Chandra modules and of primitive ideals, Invent. Math. 80 (1985), no. 1, 1-68, DOI 10.1007/BF01388547. MR784528

[BJ] Walter Borho and Jens Carsten Jantzen, Über primitive Ideale in der Einhüllenden einer halbeinfachen Lie-Algebra (German, with English summary), Invent. Math. 39 (1977), no. 1, 1-53, DOI 10.1007/BF01695950. MR0453826 
[Bou] N. Bourbaki, Éléments de mathématique. Fasc. XXXIV. Groupes et algèbres de Lie. Chapitre IV: Groupes de Coxeter et systèmes de Tits. Chapitre V: Groupes engendrés par des réflexions. Chapitre VI: systèmes de racines (French), Actualités Scientifiques et Industrielles, No. 1337, Hermann, Paris, 1968. MR.0240238

[Br] Jonathan Brown, Representation theory of rectangular finite $W$-algebras, J. Algebra 340 (2011), 114-150, DOI 10.1016/j.jalgebra.2011.05.014. MR2813566

[BrG] Jonathan S. Brown and Simon M. Goodwin, Finite dimensional irreducible representations of finite $W$-algebras associated to even multiplicity nilpotent orbits in classical Lie algebras, Math. Z. 273 (2013), no. 1-2, 123-160, DOI 10.1007/s00209-012-0998-8. MR.3010154

[BK] Jonathan Brundan and Alexander Kleshchev, Schur-Weyl duality for higher levels, Selecta Math. (N.S.) 14 (2008), no. 1, 1-57, DOI 10.1007/s00029-008-0059-7. MR2480709

[CM] David H. Collingwood and William M. McGovern, Nilpotent orbits in semisimple Lie algebras, Van Nostrand Reinhold Mathematics Series, Van Nostrand Reinhold Co., New York, 1993. MR 1251060

[Dodd] Christopher Dodd, Injectivity of the cycle map for finite-dimensional $W$-algebras, Int. Math. Res. Not. IMRN 19 (2014), 5398-5436, DOI 10.1093/imrn/rnt106. MR.3267375

[Dou] J. Matthew Douglass, Cells and the reflection representation of Weyl groups and Hecke algebras, Trans. Amer. Math. Soc. 318 (1990), no. 1, 373-399, DOI 10.2307/2001244. MR 1035211

[GS] Dimitar Grantcharov and Vera Serganova, Cuspidal representations of $\mathfrak{s l}(n+1)$, Adv. Math. 224 (2010), no. 4, 1517-1547, DOI 10.1016/j.aim.2009.12.024. MR2646303

[Dyn] E. B. Dynkin, Semisimple subalgebras of semisimple Lie algebras (Russian), Mat. Sbornik N.S. 30(72) (1952), 349-462 (3 plates). MR0047629

[HK] Ruth Stella Huerfano and Mikhail Khovanov, A category for the adjoint representation, J. Algebra 246 (2001), no. 2, 514-542, DOI 10.1006/jabr.2001.8962. MR1872113

[Hum] James E. Humphreys, Representations of semisimple Lie algebras in the BGG category $\mathcal{O}$, Graduate Studies in Mathematics, vol. 94, American Mathematical Society, Providence, RI, 2008. MR 2428237

[KZ] A. W. Knapp and Gregg J. Zuckerman, Classification of irreducible tempered representations of semisimple groups. II, Ann. of Math. (2) 116 (1982), no. 3, 457-501, DOI 10.2307/2007019. MR678478

[Jo1] Anthony Joseph, On the associated variety of a primitive ideal, J. Algebra 93 (1985), no. 2, 509-523, DOI 10.1016/0021-8693(85)90172-3. MR786766

[Jo2] Anthony Joseph, Orbital varietes of the minimal orbit (English, with English and French summaries), Ann. Sci. École Norm. Sup. (4) 31 (1998), no. 1, 17-45, DOI 10.1016/S00129593(98)80017-7. MR.1604290

[Kos] Bertram Kostant, On Whittaker vectors and representation theory, Invent. Math. 48 (1978), no. 2, 101-184, DOI 10.1007/BF01390249. MR507800

[LO] Ivan Losev and Victor Ostrik, Classification of finite-dimensional irreducible modules over $W$-algebras, Compos. Math. 150 (2014), no. 6, 1024-1076, DOI 10.1112/S0010437X13007604. MR.3223881

[Los1] Ivan Losev, Finite-dimensional representations of W-algebras, Duke Math. J. 159 (2011), no. 1, 99-143, DOI 10.1215/00127094-1384800. MR2817650

[Los2] Ivan Losev, Quantized symplectic actions and $W$-algebras, J. Amer. Math. Soc. 23 (2010), no. 1, 35-59, DOI 10.1090/S0894-0347-09-00648-1. MR2552248

[Lyn] Thomas Emile Lynch, Generalized Whittaker vectors and representation theory, ProQuest LLC, Ann Arbor, MI, 1979. Thesis (Ph.D.)-Massachusetts Institute of Technology. MR2940885

[Mit] Barry Mitchell, Theory of categories, Pure and Applied Mathematics, Vol. XVII, Academic Press, New York-London, 1965. MR0202787

[Pet] Alexey Petukhov, On the Gelfand-Kirillov conjecture for the W-algebras attached to the minimal nilpotent orbits, J. Algebra 470 (2017), 289-299, DOI 10.1016/j.jalgebra.2016.08.021. MR.3565435

[Pr1] Alexander Premet, Special transverse slices and their enveloping algebras, Adv. Math. 170 (2002), no. 1, 1-55, DOI 10.1006/aima.2001.2063. With an appendix by Serge Skryabin. MR.1929302 
[Pr2] Alexander Premet, Enveloping algebras of Slodowy slices and the Joseph ideal, J. Eur. Math. Soc. (JEMS) 9 (2007), no. 3, 487-543, DOI 10.4171/JEMS/86. MR2314105

[PT] Alexander Premet and Lewis Topley, Derived subalgebras of centralisers and finite $W$ algebras, Compos. Math. 150 (2014), no. 9, 1485-1548, DOI 10.1112/S0010437X13007823. MR.3260140

[Vog] David A. Vogan Jr., A generalized $\tau$-invariant for the primitive spectrum of a semisimple Lie algebra, Math. Ann. 242 (1979), no. 3, 209-224, DOI 10.1007/BF01420727. MR545215

The University of Manchester, Oxford Road M13 9PL, Manchester, United Kingdom; and Institute for Information Transmission Problems, Bolshoy Karetniy 19-1, Moscow 127994, Russia

Email address: alex--2@yandex.ru 